\title{
PLASMA PRODUCED BY A LASER IN A MEDIUM WITH CONVECTION AND FREE SURFACE SATISFYING A HAMILTON-JACOBI EQUATION
}

\author{
W. ECKHAUS*, A. VAN HARTEN ${ }^{*}$ and Z. PERADZYŃSKI ${ }^{\ddagger}$ \\ * Department of Mathematics, State University of Utrecht, 3512 LR Utrecht. The Netherlands \\ ‡Institute for Fundamental Technological Research, Warsaw, Poland
}

Received 27 March 1986

Revised manuscript received 12 December 1986

\begin{abstract}
A nonlinear diffusion equation is considered which models the temperature distribution in a laser-sustained plasma subject to wind. As certain parameters are small a singular perturbation problem arises and the method of matched asymptotic expansions is applied to approximate the solution. An essential role in this problem is played by the plasma front. This is a free surface separating the plasma and non-plasma phases. One of the main results is that we derive a 1 st order nonlinear P.D.E. of Hamilton-Jacobi type, which describes certain parts of the free surface in the stationary case. This equation is analysed for various wind directions. It appears that for the initial conditions for these parts of this free surface there are different possibilities depending on the wind direction.

We show further that the solution of the Hamilton-Jacobi equation can contain singularities of corner type. Furthermore, the effect of wind on the stability region of the stationary full plasma solution is analysed. The method of analysis presented in this paper is not restricted to the cone-like geometry or the specific form of the non-linearity of the problem considered here, but has potentially a much wider scope. However, the case under study in this paper is certainly representative for the effects that have to be taken into account.
\end{abstract}

\section{Introduction}

In this paper we continue, from [1], the study of nonlinear singularly perturbed free boundary problems which can be considered as a model for the functioning of an experimental apparatus to maintain plasma by focussing a laser beam. These problems lead to highly interesting mathematical phenomena. Let us first describe the physical situation. In a chamber with pressurized gas a focussing devise produces a rotationally symmetric focussed laser beam. By ignition a region of plasma can be created near the focal point and maintained under a suitable combination of parameters such as pressure and laser intensity. The situation is sketched in fig. 1 .

In the first part of our study [1] we have taken $V=0$. The forward and backward free boundaries of the plasma are then rotationally symmetrical. We have also restricted the analysis to the region inside the light cone, away from its boundary.
In the present study we include the analysis of the transition layers near the boundary of the light cone and furthermore we admit a (homogeneous) windfield with $V \neq 0$ and an arbitrary orientation with respect to the apparatus. The effect of the windfield is mathematically highly interesting: the forward and backward free boundaries are defined as solutions of Hamilton-Jacobi equations for which suitable initial condition on the (unknown) intersection with the lightcone must be found. Let us now briefly describe the mathematical modelling. As in [1] we consider a simplified energy balance in terms of the temperature $T$, i.e. an equation of the structure

$c\left(\frac{\partial T}{\partial t}+V \cdot \nabla T\right)=\operatorname{div}(\sigma \nabla T)-\Gamma+Q$.

On the right-hand side the first term represents thermal diffusion, $-\Gamma$ are heat losses due to radiation and $Q$ is the absorption of energy from the laser beam. 


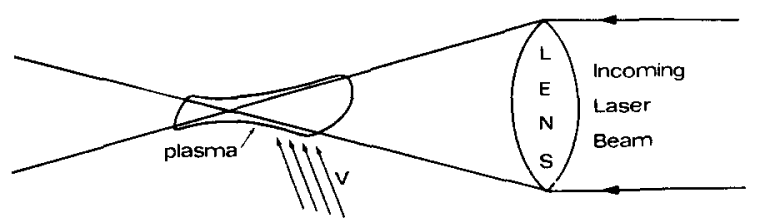

Fig. 1. In this figure $V$ symbolizes the flow field of gas, which can be either forced on purpose in the experimental apparatus, or simply induced by buoyancy forces due to the gravitational field.

In this paper we take $\sigma=\sigma_{0}$ constant, furthermore

$\Gamma=\gamma_{0}\left(T-T_{0}\right) H\left(T-T_{0}\right)$,

where $H$ is the Heaviside unit step function and $\gamma_{0}$ another constant. The absorption term will be described by

$Q=\kappa(T) I(x)$,

where $\kappa(T)$ is the absorption coefficient and $I(x)$ the intensity of the laser light at a point $x$.

Let $\|x\|$ be the distance from the theoretical focal point to the point $x$. By $l(x)$ we denote the part of the light ray through $x$ at the right of $x$. As in [1] we shall use the following formula:

$I(x)=I_{0} \frac{J(x)}{\|x\|^{2}+a^{2}} \exp \left\{-\int_{l(x)} \kappa(T) \mathrm{d} \xi\right\}$.

$I_{0}$ measures the intensity of the incoming laser beam, $J(x)$ is responsible for distribution of intensity over angular variables, $\left(\|x\|^{2}+a^{2}\right)^{-1}$ models the effect of focussing, with $a^{2}$ the imperfection of the focussing device, and the exponential function accounts for loss of intensity due to absorption.

The final observation is that the absorption coefficient is virtually zero for $T<T_{1}$ and rises sharply to a near-constant value for $T>T_{1}$. This will be modelled by

$\kappa(T)=\kappa_{0} H\left(T-T_{1}\right)$.

One now introduces non-dimensional quantities described in [1] and obtains as mathematical problem the equation

$$
\begin{aligned}
& \frac{\partial u}{\partial t}+\delta w \cdot \nabla u \\
& \quad=\varepsilon^{2} \Delta u+\frac{m}{\|x\|^{2}+a^{2}} \mathrm{e}^{-d} H(u-1)-u
\end{aligned}
$$

In that equation $d=\int_{/(x)} H(u-1) \mathrm{d} \xi$, i.e. for $x$ inside the plasma $d$ is the distance from the point $x$ to the right boundary of the plasma measured along a lightray. Furthermore, the parameters are defined through the formulas

$$
\begin{aligned}
& \varepsilon^{2}=\frac{\sigma_{0} \kappa_{0}^{2}}{\gamma_{0}}, \quad m=\frac{I_{0} \kappa_{0}^{3}}{\gamma_{0}\left(T_{0}-T_{1}\right)}, \\
& \delta=\frac{c|V|}{\sigma_{0} \kappa_{0} \gamma_{0}}, \quad w=\frac{V}{|V|} .
\end{aligned}
$$

$\varepsilon$ is considered as a small parameter, so that (1.6) is a problem of singular perturbation. With respect to $\delta$ some considerations are needed. We are going to use methods of singular perturbations, cf. [2], in which a proper choice of $\delta$ with respect to $\varepsilon$ must be made. We wish to make this choice such that

1) there are non-trivial solutions for $\varepsilon \downarrow 0$;

2) the effect of wind is already present in the first approximation.

The first of the conditions simply means that we do not wish the plasma to be blown away by wind. We find, and this will be confirmed in the sequel, that the proper choice is

$\delta=\varepsilon$.

With respect to the function $J$, which describes the distribution of intensity of the laser beam over angular variables we shall use the following representation:

$J= \begin{cases}0, & \text { outside light cone } \\ 1-\exp \left(-\frac{\eta}{\|x\| b}\right), & \text { inside light cone }\end{cases}$

where $\eta$ denotes the distance to the light cone and 
$b$ is another (positive) small parameter. Thus light is nearly uniform within the cone and falls off rapidly to zero near the boundary of the cone.

We relate $b$ to $\varepsilon$ by requiring $\varepsilon \ll b \ll 1$. From now on we are going to analyse stationary solutions $u(x)$ of $(1.6)$, i.e.

$$
\begin{aligned}
& \varepsilon^{2} \Delta u-\varepsilon w \cdot \nabla u \\
& \quad+\frac{m}{\|x\|^{2}+a^{2}} J \mathrm{e}^{-d} H(u-1)-u=0,
\end{aligned}
$$

with the condition $u \rightarrow 0$ as $\|x\| \rightarrow \infty$. The central problem is the construction of the free boundary on which $u=1$.

\section{Asymptotic approximation of a stationary solution}

Let us consider the construction of an asymptotic approximation for $\varepsilon \downarrow 0$ of a stationary solution of (1.10) of "full plasma type" analogous to ref. 1 , section 2 . In the plane determined by the wind vector $w$ and the central axis of the cone the profile of the plasma and the layer structure can typically be of the shape sketched in fig. 2 .

In this figure the wind makes an angle $\alpha, 0 \leq \alpha$ $\leq \pi$ with the negative central axis. In general one should expect that the plasma profile will depend heavily on the direction of the wind. An interesting question is how the direction of the wind influences the plasma profile. Later on we shall see that in this respect we need to distinguish between several cases.

Inside the plasma, $\mathcal{O}(1)$ away from the plasma boundary, our approximation is of a regular type and its zeroth order term is given by

$$
U_{0}(r, \theta, \phi)=\frac{m J}{r^{2}+a^{2}} \exp \left(r-R_{0}(\theta, \phi)\right),
$$

where $r=R_{0}(\theta, \phi)>0$ is the 0 th order approximation of the surface at the front, which is still unknown. For the coordinates $r, \theta, \phi$ we use the following convention. Inside the right part of the cone $r, \theta, \phi$ are spherical coordinates where for a point $\boldsymbol{x}=(x, y, z), r=\|x\|>0, \theta=\arcsin (z / r)$ and $\phi=\arctan (y / x)$. In the left part of the cone we define for a point $\boldsymbol{x}: r=-\|\boldsymbol{x}\|<0$ and $\theta$ and $\phi$ are the spherical angles corresponding to $-\boldsymbol{x}$. Outside the plasma, $\mathcal{O}(1)$ away from the plasma boundary, $U_{0}=0$.

In order to get more information about the position of the free surface at the front we study the free surface layer there. This can be done along similar lines as in [1]. Let the position of the free surface $S_{0}$ be given by $\Psi_{0}(x)=0$ with $\nabla \Psi_{0} \neq$ 0 and $\Psi_{0}<0$ inside the plasma. In spherical coordinates one can take $\Psi_{0}=r-R_{0}(\theta, \phi)$. The coordinate transversal to the free surface is stretched in the following way:

$\xi=\Psi_{0}\left\|\nabla \Psi_{0}\right\|^{-1} \varepsilon^{-1}$

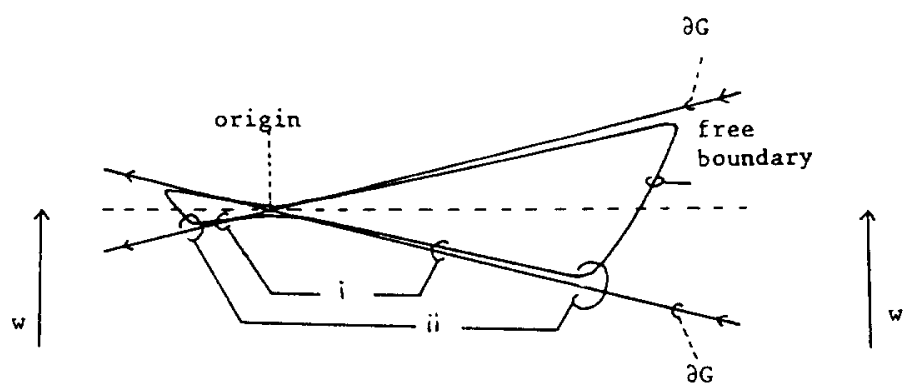

Fig. 2. i) The rigid part of the free boundary, i.e. the free boundary follows the boundary of the lightcone, $\partial G$, up to $\mathcal{O}(b)$. ii) Separation of the free boundary from the rigid one 
Note, that $\xi<0$ inside the plasma and $\xi>0$ outside the plasma.

For the 0th order layer term $G_{0}$ we find the following O.D.E.:

$\frac{\mathrm{d}^{2} G_{0}}{\mathrm{~d} \xi^{2}}-(w \cdot n) \frac{\mathrm{d} G_{0}}{\mathrm{~d} \xi}-G_{0}=F H(\xi)$,

with $F=\left.U_{0}\right|_{s_{0}}=m J /\left(R_{0}(\theta, \phi)^{2}+a^{2}\right)$ and $n$ the normal on $S_{0}$ in outward direction from the plasma region. Solving this equation, taking into account matching with the regular expansion $U_{0}$ and the condition $G_{0}=1$ on $S_{0}$, we obtain

$G_{0}= \begin{cases}\exp \left(\lambda_{-} \xi\right) & \text { for } \xi \geq 0 \\ F+(1-F) \exp \left(\lambda_{+} \xi\right) & \text { for } \xi \leq 0\end{cases}$

with $\lambda_{ \pm}=\frac{1}{2}\left\{w \cdot n \pm \sqrt{(w \cdot n)^{2}+4}\right\}$. Since there is no heat source of $\delta$ function type on $S_{0}$ the derivative of $G_{0}$ should be continuous at $S_{0}$. This leads us to the condition

$F=\frac{2}{1+q / \sqrt{q^{2}+4}}, \quad$ with $q=w \cdot n$.

The unknown surface $S_{0}$ has to satisfy this first order nonlinear P.D.E. of the Hamilton-Jacobi type: the inner product $w \cdot n$ with $n$ the normal to the surface has a prescribed value, cf. [3]. Representing $S_{0}$ by zeros of some function $\psi(x)$, and expressing $n$ in terms of $\nabla \psi, n=\|\nabla \psi\|^{-1} \nabla \psi$, one arrives at the nonlinear equation for $\psi$. The surface $S_{0}$ satisfying eq. (2.2) will be referred to as Hamilton-Jacobi surface or simply $\mathrm{H}-\mathrm{J}$ surface. Of course eq. (2.2) in itself is not sufficient to determine the surface $S_{0}$. One needs more information, for example initial conditions. These can be provided for instance by the knowledge of the curve on $\partial G$, where the separation of the free boundary from the rigid boundary takes place.

A first reaction is that this requires a detailed analysis of the rather complicated corner layer where the surface $S_{0}$ meets the boundary of the cone and indeed this is so.
However, we found a nice, rather simple argument that in certain cases of the wind direction provides us with a "start curve" on the cone for this Hamilton-Jacobi surface. In some other cases the surface "starts" from what we call a critical point. This will be shown in the next section. There arises however another difficulty as we shall see later on. One cannot always expect that a nonlinear equation as eq. (2.2) has smooth global solutions. The surface we are looking for and which can be represented locally by $\psi(x)=0$, might have singularities, for example self-intersections, thus forming solutions with angles, i.e. "corners". Admitting corners we enlarge the class of possible solutions enormously. On the other hand in the vicinity of a corner eq. (2.2) is no longer valid and using additional reasoning based on the Green function of a linear part of eq. (1.10) we are able to select admissible corners and reject inadmissible ones. This in turn reduces the number of possible solutions to just a few, see section 3.1.

In the first part of section 3 we shall discuss as an illuminating exercise the two dimensional problem in the plane through $w$ and the central axis. Because of symmetry the intersection of $S_{0}$ and this plane can be found independently to a certain degree and the reasoning is somewhat simpler in this case. Next we deal with the full three-dimensional problem in section 3.3. Note that in (2.2) we still allow a general distribution $J$ over the angles. From now on we specialize to the case given in (1.9).

For the free surface at the back $r=-\tilde{R}_{0}(\theta, \phi)$ $<0$ a similar equation as (2.2) can be derived

$\tilde{F}=\frac{2}{1+\tilde{q} / \sqrt{\tilde{q}^{2}+4}}$

where $\tilde{q}=w \cdot \tilde{n}$ with $\tilde{n}$ the outward normal on the surface and $\tilde{F}$ is the reduced intensity

$\tilde{F}=\frac{\exp \left\{-\left(R_{0}(\theta, \phi)+\tilde{R}_{0}(\theta, \phi)\right)\right\}}{\tilde{R}_{0}(\theta, \phi)^{2}+a^{2}}$. 
It is clear that for the free surface at the back knowledge on the free surface at the front is needed as input. Furthermore, in addition to (2.3) extra information is necessary to start with the surface analogously to the situation of the free surface at the front.

Again we refer to section 3 for further details. Once the free surfaces $r=R_{0}(\theta, \phi)$ and $r=$ $-\tilde{R}_{0}(\theta, \phi)$ have been found the region where the regular approximation holds is known. The construction is consistent only if the condition $U_{0}>1$ is not violated in the plasma region. This has to be checked, see section 4 .

Let us now consider the layers associated with the rigid part of the free boundary. As mentioned above these parts of the boundary are staying close, within $\mathcal{O}(b)$ accuracy, to the boundary of $G$.

To start with we consider the free surface near the positive part of the boundary of the cone, $\partial G_{+}$. Let the position of the free surface be given by

$\eta=b \cdot g_{0}(r, \chi)+\cdots$,

with $\eta$ the distance to the cone and $\chi$ the angle around the cone with $\chi=0 \leftrightarrow y=0, \theta<0$ and $x>0$ for $y>0$. Note, that in the first order in $b$ the outward normal to this surface is equal to $\nu(\chi)$ the outward normal on $\partial G_{+}$.

Analysis of the layer of $\varepsilon$-width along this surface, completely analogous to what we did before, provides us with an algebraic equation for $g_{0}$,

$\frac{m}{r^{2}+a^{2}}\left[1-\exp \left(-\frac{g_{0}}{r}\right)\right]=2 /\left(1+\beta_{c}(\chi)\right)$

with $\beta_{c}=w \cdot \nu / \sqrt{4+(w \cdot \nu)^{2}}$. Note that this equation can be solved only if $r$ is sufficiently small,

$r<\rho(\chi)$, where $\rho$ is found from the condition

$m /\left(\rho^{2}+a^{2}\right)=2 /\left(1+\beta_{c}(\chi)\right)$, i.e.

$\rho(\chi)=\left\{\frac{1}{2} m\left(1+\beta_{\mathrm{c}}(\chi)\right)-a^{2}\right\}^{1 / 2}$.

Of course, the separation of the free surface from the rigid part along $\partial G_{+}$is possible only if (2.6) is still satisfied. Therefore (2.6) is a restriction for the location of the curve $\gamma=L(\chi)$ on $\partial G_{+}$, where the separation takes place. When the separation takes place on a scale of size $b$ it is also possible to analyse the behaviour of the free surface near such a transition curve $r=L(\chi)$. We put

$\eta=b \cdot h(\zeta, \chi)+\ldots$

with $\zeta=(r-L(\chi)) / b$.

As $b$ defines a scale which is larger then the $\varepsilon$-scale, i.e. $\varepsilon / b \ll 1$, it still makes sense to introduce such layer behaviour even though its curvature is of order $O(1 / b)$.

Analysis of the layer of $\varepsilon$-width now yields an O.D.E. for $h$ with respect to the variable $\zeta$

$$
\begin{aligned}
w \cdot n & =\frac{w_{\nu}-\left\{w_{r}-w_{\chi} \frac{\mathrm{d} L}{\mathrm{~d} \chi}\right\} \frac{\mathrm{d} h}{\mathrm{~d} \zeta}}{\sqrt{1+\left(1+\left(\frac{\mathrm{d} L}{\mathrm{~d} \chi}\right)^{2}\right)\left(\frac{\mathrm{d} h}{\mathrm{~d} \zeta}\right)^{2}}} \\
& =\frac{2 \beta^{\prime}}{\sqrt{1-\beta^{\prime 2}}},
\end{aligned}
$$

with $w_{\nu}, w_{r}, w_{\chi}$ the components of the wind in the direction of $\nu$, in radial direction and in the $\chi$-angular direction, respectively, and

$\beta^{\prime}=\frac{2}{m}\left(r^{2}+a^{2}\right) /\left(1-\exp \left(-\frac{h}{L(\chi)}\right)\right)-1$.

Suppose that the curve $r=L(\chi)$ is known. Eq. (2.8) has to be solved with the starting value for $\zeta \rightarrow-\infty$ implied by the matching with the solution of $(2.5)$ i.e. $h(-\infty, \chi)$. For $\zeta \rightarrow \infty$ it is required that $\mathrm{d} h / \mathrm{d} \zeta$ tends to a constant which agrees with eq. (2.2) or its equivalent form (3.1). As it follows from (2.8) this is the case provided $h$ 


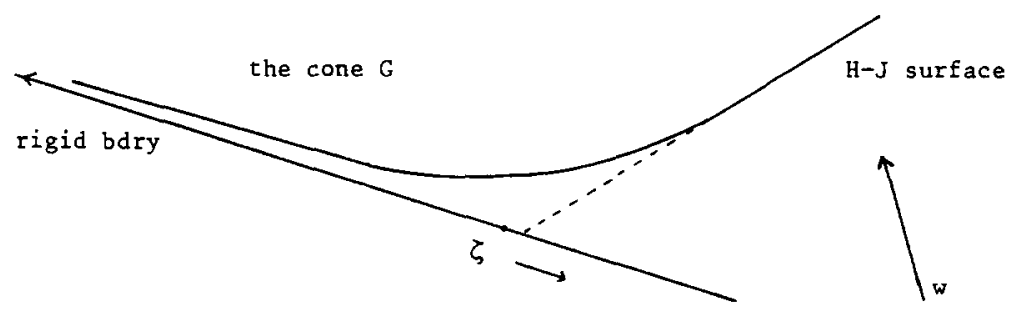

Fig. 3.

tends simultaneously to $+\infty$. If the separation takes place at the last possible position $r=\rho(\chi)$ it is easily seen that the $\mathrm{H}-\mathrm{J}$ surface has to be tangent to the boundary of the cone (see fig. 3).

In this way the matching of the rigid boundary with this part of the free boundary which comes from the interior of the cone $G$ can be done. However the transition is not always possible. As a matter of fact the right-hand side of eq. (2.8) is decreasing with $h$. Therefore, in order to assure the existence of such a transistion of the rigid boundary to the $H-J$ surface it is necessary that $w \cdot n$ is not increasing as $\zeta$ increases.

It appears that this condition imposes certain restrictions on the curve $r=L(\chi)$. Together with other conditions of a global nature this will determine this curve completely, as we shall see. The rigid boundary as described by (2.4) and (2.5) together with the part of boundary defined by (2.8) can be viewed as the limiting cases of $\mathrm{H}-\mathrm{J}$ surfaces for $b=\mathcal{O}(1)$ becoming small. Therefore it may happen that within the $\mathcal{O}(b)$ layer near $\partial G$ admissible corners will appear. When the distribution of the intensity is given by (1.9) this can happen only in cases where the mentioned monotonicity property is violated. Instead of having one branch we have the possibility of two branches satisfying (2.9) forming an angle. One of these branches is matching with the rigid part of the plasma front and the other one with the $\mathrm{H}-\mathrm{J}$ part of the free surface. If this occurs one has to decide on the basis of the analysis in section 3 whether the corner is admissible. It can easily be checked that if the monotonicity property is not violated such an admissible corner is just impossible.
Near the negative part of the boundary cone $\partial G_{-}$the free surface can be analysed in an analogous way. It leads to the conclusion that the surface can only stay near $\partial G_{-}$as long as the condition

$\|x\|<\tilde{\rho}(\chi)$

is satisfied. Here $\chi$ is the angle of $-x$ as introduced in (2.4), i.e. points on the same ray have the same $\chi$ angle and $\tilde{\rho}$ is defined implicitly by

$\bar{\rho}=\left\{\frac{1}{2} \tilde{m}(\chi, \rho)(1+\tilde{\beta}(\chi))-a^{2}\right\}^{1 / 2}$,

$\tilde{\beta}=\frac{w \cdot \tilde{\nu}}{\sqrt{4+(w \cdot \tilde{\nu})^{2}}}, \quad \tilde{v}(\chi)=-\nu(\chi)$,

$\tilde{m}(\chi)=m \exp (-\tilde{\rho}-L(\chi))$,

with $L(\chi)$ the curve where the right free surface meets $\partial G_{+}$. If this condition is satisfied the position is given by a formula analogous to (2.5). The transition to the left free surface of HamiltonJacobi type can be described analogously to (2.8) and again the conclusion is that at the maximal position $\|x\|=\tilde{\rho}(\chi)$ the Hamilton-Jacobi surface has to start tangently to $\partial G$.

Let us conclude this section with a few remarks about the layer at the origin of size $\varepsilon \times \varepsilon \times \varepsilon$. Once $R_{0}(\theta, \phi)$ has been found the intensity near the origin is known. In the case $\varepsilon \ll a$ and $a=\mathcal{O}(1)$, the behaviour in this neighbourhood is approximately given by

$u \simeq \frac{1}{a^{2}} U_{\text {or }}\left(\frac{x}{\varepsilon}\right)+\cdots$. 
Here $U_{\text {or }}$ has to satisfy

$(\Delta-w \cdot \nabla-1) U=-m \exp \left(-R_{0}(\theta, \Phi)\right) \cdot I_{\text {cone }}$,

with $I_{\text {cone }}$ the indicator function of the cone. It is left to the reader to give the solution of (2.12) in terms of the Green's kernel of the equation (compare (3.1.3)). The other cases $\varepsilon \sim a \ll 1$ or $a \ll$ $\varepsilon \ll 1$ can be dealt with in an analogous way.

\section{Free surface determined by an equation of Hamilton-Jacobi type}

The equation for the free surface at the front derived in (2.2) can be reformulated as

$w \cdot n=2 \beta / \sqrt{1-\beta^{2}}$

with $n$ the outward normal on the plasma region and $\beta=(2 / m)\left(\|x\|^{2}+a^{2}\right)-1$. An equivalent formulation in terms of the function $\Psi_{0}$ is

$\frac{w}{\|w\|} \nabla \Psi_{0}=\mu\left\|\nabla \Psi_{0}\right\|$

where $\mu \stackrel{\text { def }}{=} 2 \beta /\left(\|w\| \sqrt{1-\beta^{2}}\right)$. Note that in the case of $J$ given by (1.9) $\mu$ depends only $r=\|x\|$. An immediate observation is, that the equation can only have solutions in the region where $|\mu| \leq 1$, i.e. $-\hat{\beta} \leq \beta \leq \hat{\beta}$ with $\hat{\beta}=\|w\| / \sqrt{4+\|w\|^{2}}$ and

$\rho_{-} \leq\|x\| \leq \rho_{+}$,

with

$\rho_{ \pm}=\sqrt{\frac{m}{2}(1 \pm \hat{\beta})-a^{2}}$.

The wind vector can only be perpendicular to the unknown free surface for $\|x\|=\rho_{-}(w=-\|w\| \cdot n)$ or $\|x\|=\rho_{+}(w=\|w\| \cdot n)$. At the special position $r=\rho_{0} \stackrel{\text { def }}{=} \sqrt{(m / 2)-a^{2}}$, which corresponds to the plasma front in the case without wind, the wind vector has to be tangent to the unknown free
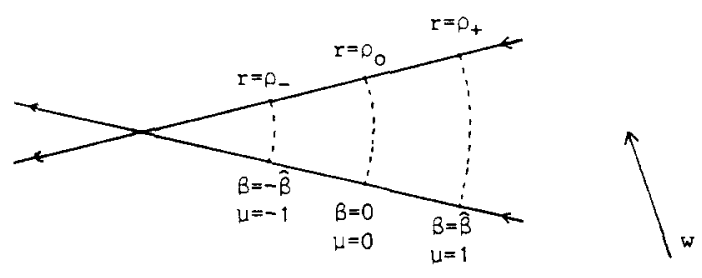

Fig. 4.

surface. Fig. 4 summarizes this situation in the plane of symmetry.

Let us first discuss briefly some general theory concerning first order P.D.E. such as (3.1-2). In order to find a surface $\Psi_{0}=0$ satisfying eqs. (3.1-2) one can use the method of characteristic curves. Let us introduce the notation $p=\nabla \Psi_{0}$ and define a Hamiltonian $H(x, p)$ by

$H(x, p)=-\frac{w}{\|w\|} \cdot p+\mu\|p\|$.

A characteristic curve $x(t)$ in combination with $p(t)=\nabla \Psi_{0}(x(t))$ can be found by solving the Hamiltonian system

$$
\begin{aligned}
& \frac{\mathrm{d} x}{\mathrm{~d} t}=-\frac{\partial H}{\partial p}=\frac{w}{\|w\|}-\mu \frac{p}{\|p\|}, \\
& \frac{\mathrm{d} p}{\mathrm{~d} t}=\frac{\partial H}{\partial x}=\|p\| \frac{\mathrm{d} \mu}{\mathrm{d} r} \cdot \frac{x}{r},
\end{aligned}
$$

in combination with suitable initial conditions. Suppose initially a $C^{1}$ curve parametrized with $s$ for the position $x$ is given as $x_{0}(s)$ with $\mathrm{d} x_{0} / \mathrm{d} s$ $\neq 0$. Initial conditions $p_{0}(s)$ for the variable $p$ must be determined such that $p_{0}(s)$ has an outward direction with respect to the plasma and

$p_{0}(s) \frac{\mathrm{d} x_{0}}{\mathrm{~d} s}(s)=0$,

$H\left(x_{0}(s), p_{0}(s)\right)=0$.

Interpreting the conditions in (3.6) as the intersection of a plane perpendicular to $\mathrm{d} x_{0} / \mathrm{d} s$ with a half-cone, a simple calculation shows that 
nontrivial solutions $p_{0}(s)$ can only be found if

$\left(\frac{w}{\|w\|} \frac{\mathrm{d} x_{0}}{\mathrm{~d} s}\right)^{2} \leq\left\|\frac{\mathrm{d} x_{0}}{\mathrm{~d} s}\right\|^{2}\left(1-\mu^{2}\right)$.

It is not difficult to verify, that the equality

$$
\left(\frac{w}{\|w\|} \cdot \frac{\mathrm{d} x_{0}}{\mathrm{~d} s}\right)^{2}=\left\|\frac{\mathrm{d} x_{0}}{\mathrm{~d} s}\right\|^{2}\left(1-\mu^{2}\right) \quad \text { at } x_{0}\left(s_{0}\right)
$$

will precisely be satisfied in the situation that $x_{0}(s)$ has a semi-characteristic direction at $x_{0}\left(s_{0}\right)$ in the sense that the vectors $\gamma=\mathrm{d} x_{0} / \mathrm{d} s\left(s_{0}\right)$ and $v=\mathrm{d} x /\left.\mathrm{d} t\right|_{t=0, s=s_{0}}$ are linearly dependent. The condition for linear dependence $(\gamma \cdot v)^{2}=\|\gamma\|^{2}\|v\|^{2}$ reduces to $(3.7)_{\mathrm{ch}}$ because of $(3.5-6)$. In a noncharacteristic case with

$\left(\frac{w}{\|w\|} \cdot \frac{\mathrm{d} x_{0}}{\mathrm{~d} s}\right)^{2}<\left\|\frac{\mathrm{d} x_{0}}{\mathrm{~d} s}\right\|^{2}\left(1-\mu^{2}\right) \quad$ at $x_{0}\left(s_{0}\right)$,

two possible choices for $p\left(s_{0}\right) /\left\|p\left(s_{0}\right)\right\|$ arise. The case $(3.7)_{\mathrm{ch}}$ is also special because these two possibilities for $p\left(s_{0}\right) /\left\|p\left(s_{0}\right)\right\|$ coincide and are given by

$\frac{p_{0}}{\left\|p_{0}\right\|}=\frac{1}{\mu}\left\{\frac{w}{\|w\|}-\alpha\left\|\frac{\mathrm{d} x_{0}}{\mathrm{~d} s}\right\|^{-1} \frac{\mathrm{d} x_{0}}{\mathrm{~d} s}\right\}$

with $\alpha=\left(w \cdot \mathrm{d} x_{0} / \mathrm{d} s\right) /\left(\|w\| \cdot\left\|\mathrm{d} x_{0} / \mathrm{d} s\right\|\right), \quad \alpha^{2}=$ $1-\mu^{2}$. Note, that $p_{0} /\left\|p_{0}\right\|$ is then proportional to the component of the wind perpendicular to $x_{0}(s)$ at $x_{0}\left(s_{0}\right)$. After satisfying (3.6) freedom remains in the choice of $\left\|p_{0}(s)\right\|$. This corresponds to the property that changing the initial conditions $\left(x_{0}(s), p_{0}(s)\right)$ to $\left(x_{0}(s), \lambda(s) p_{0}(s)\right)$ with $\lambda(s)>0$ has the effect that the solution $x(t, s), p(t, s)$ changes into $x(t, s), \lambda(s) p(t, s)$. This freedom in $\left\|p_{0}(s)\right\|$ can be exploited to one's convenience, for example by normalizing $\left\|p_{0}(s)\right\|=1$.

In the semi-characteristic case $(3.7)_{\mathrm{ch}}$ it can be used to ensure that $\mathrm{d} p_{0} / \mathrm{d} s$ and $\mathrm{d} p /\left.\mathrm{d} t\right|_{t=0}$ are linearly independent, i.e.

$$
\left(\frac{\mathrm{d} p_{0}}{\mathrm{~d} s} \cdot \frac{x_{0}}{\left\|x_{0}\right\|}\right)^{2}<\left\|\frac{\mathrm{d} p_{0}}{\mathrm{~d} s}\right\|^{2} .
$$

If $p_{0}(s) \cdot x_{0}(s) \neq 0$ one can even arrange that $\left(\mathrm{d} p_{0} / \mathrm{d} s\right) \cdot x_{0}=0, \mathrm{~d} p_{0} / \mathrm{d} s \neq 0$. Let us now suppose that $p_{0}(s)$ is non-zero and $s$-dependent in a $\mathrm{C}^{1}$ way, while $(3.7)_{\mathrm{nch}}$ or $(3.7)_{\mathrm{ch}}$ and (3.8) are satisfied. From the theory of O.D.E.'s it follows that (3.5) together with the initial conditions has a uniquely defined solution $x(t, s), p(t, s)$. This solution is $C^{1}$ in $s$ and $t$ and moreover $\partial\left(\begin{array}{l}x \\ p\end{array}\right) / \partial t$ and $\partial\left(\begin{array}{l}x \\ p\end{array}\right) / \partial s$ are linearly independent. Thus this solution represents a two-dimensional $\mathrm{C}^{1}$ variety in $\mathbb{R}^{6}$, the $(x, p)$-space. It is simple to check, that everywhere

$H(x(t, s), p(t, s))=0$,

$p \cdot \frac{\partial x}{\partial t}=0$ and $p \cdot \frac{\partial x}{\partial s}=0$.

Suppose that at a certain point $x\left(t_{0}, s_{0}\right)$ the vectors $\partial x\left(t_{0}, s_{0}\right) / \partial t$ and $\partial x\left(t_{0}, s_{0}\right) / \partial s$ are linearly independent and $p\left(t_{0}, s_{0}\right) \neq 0$. A standard application of the implicit function theorem then shows, that $x(t, s)$ with $t, s$ close to $t_{0}, s_{0}$ is a 2 -dim. $C^{1}$ surface in $\mathbb{R}^{3}$ which can be described as the set $\Psi(x)=0$, where $\Psi$ is $C^{1}$ and has a non-vanishing gradient. Note that $p\left(t_{0}, s_{0}\right)$ is perpendicular to the tangent space of the surface at $x\left(t_{0}, s_{0}\right)$ because of (3.9). A simple normalization is now possible such that $\nabla \Psi(x(t, s))=p(t, s)$. The function $\Psi$ obtained in this way obviously satisfies eq. (3.2).

A projection of the two-dimensional variety constructed from the Hamiltonian system in $(x, p)$-space down to the $x$-space can, in principle, give rise to singularities in the surface in $\mathbb{R}^{3}$, such as degenerate lines or points. Such singularities might occur in certain situations as we shall see in the sequel.

In order to obtain more insight in the behaviour of the characteristics the following consequences 
of (3.5) can profitably be used:

$$
\begin{aligned}
& \frac{\mathrm{d}}{\mathrm{d} t}\left(\frac{w}{\|w\|} \cdot x\right)=1-\mu^{2} \geq 0, \\
& \frac{\mathrm{d}}{\mathrm{d} t}(p \cdot x)=\|p\| \frac{\mathrm{d} \mu}{\mathrm{d} r} r>0, \\
& \frac{\mathrm{d}}{\mathrm{d} t}(p \cdot p)=\frac{2}{r}\|p\| \frac{\mathrm{d} \mu}{\mathrm{d} r} p \cdot x .
\end{aligned}
$$

The first identity shows that the characteristics $x(t, s)$ are drifting in the direction of the wind. If initially $p_{0} \cdot x_{0}>0$ then it follows from $\mathrm{b}$ and $\mathrm{c}$ that $p \cdot x>0$ and $\|p\|>0$ are strictly increasing functions of $t$.

Another interesting consequence of (3.5) is that if initially $x_{0,2}>0, p_{0,2}>0$ and $\left\|x_{0}\right\|<\rho_{0}$ the second components $x_{2}$ and $p_{2}$ will be increasing functions of $t$ as long as $\|x\|<\rho_{0}$.

Once the right free surface has been found the left free surface can be dealt with. Representing the surface as $\tilde{\Psi}_{0}=0$ where the outward normal equals $\nabla \tilde{\Psi}_{0} /\left\|\nabla \tilde{\Psi}_{0}\right\|$ eq. (2.3) becomes

$$
\frac{w}{\|w\|} \cdot \nabla \tilde{\Psi}_{0}=\tilde{\mu}\left\|\nabla \tilde{\Psi}_{0}\right\|
$$

with $\|w\| \cdot \tilde{\mu}=2 \tilde{\beta} / \sqrt{1-\tilde{\beta}^{2}}, \quad \tilde{\beta}=(2 / \tilde{m})\left(\|x\|^{2}+\right.$ $\left.a^{2}\right)-1$, and $\tilde{m}$ is the reduced intensity $\tilde{m}=$ $m \exp \left(-\left(R_{0}+\|x\|\right)\right)$. For a solution it is necessary that $|\tilde{\mu}| \leq 1$, which corresponds, for example, to a domain as sketched in fig. 5 .

Eq. (3.11) can be solved using the Hamiltonian

$\tilde{H}=-\frac{w}{\|w\|} \cdot p+\tilde{\mu}\|p\|$

which leads to the following Hamiltonian system for characteristic curves:

$$
\begin{aligned}
& \frac{\mathrm{d} x}{\mathrm{~d} t}=-\frac{\partial \tilde{H}}{\partial x}=\frac{w}{\|w\|}-\tilde{\mu} \frac{p}{\|p\|}, \\
& \frac{\mathrm{d} p}{\mathrm{~d} t}=\frac{\partial \tilde{H}}{\partial x}=\|p\| \cdot \nabla \tilde{\mu} .
\end{aligned}
$$

In addition to (3.12) initial conditions are needed. In this respect analogous remarks as in the discussion of initial conditions for (3.5) are valid, boiling down to replacing $\mu$ by $\tilde{\mu}$ in (3.6) and (3.7) and changing (3.8) to

$$
\left(\frac{\mathrm{d} p_{0}}{\mathrm{~d} s} \cdot \nabla \tilde{\mu}\right)^{2}<\left\|\frac{\mathrm{d} p_{0}}{\mathrm{~d} s}\right\|^{2}\|\nabla \tilde{\mu}\|^{2} .
$$

Moreover characteristics have a property analogous to (3.10a): $\mathrm{d}(w \cdot x) / \mathrm{d} t=\|w\|\left(1-\tilde{\mu}^{2}\right)>0$.

Till now we have had a discussion of the Hamilton-Jacobi equation and the corresponding Hamilton system. What we need now is the specification of the initial conditions. We will come back to the determination of these initial conditions in sections 3.2-3. First we will discuss some material concerning the appearance of corner-like singularities of the $\mathrm{H}-\mathrm{J}$ surfaces.

\subsection{Corner-like singularities of the $H-J$ surface}

In this section we derive the rule of selection of what we call admissible corners out of all other corners that can be formed by two pieces of $\mathrm{H}_{-} \mathrm{J}$ surface. In order to do this we will use the integral representation of eq. (1.2)

$$
u\left(x_{0}\right)=\int_{\Omega_{u}} G\left(x-x_{0}\right) F(x) \mathrm{d} x
$$

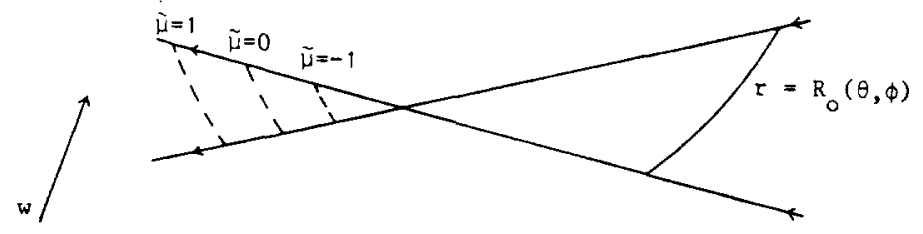

Fig. 5. 
where $\Omega_{u}=\left\{x \in \mathbb{R}^{3} ; u(x) \geq 1\right\}, F(x)$ denotes the local intensity and where $G(y)$, the Green kernel of $\varepsilon w \nabla u-\varepsilon^{2} \Delta u-u$, is given by

$$
\begin{aligned}
G(y)= & \frac{\sqrt{w^{2}+4}}{8 \pi \varepsilon^{2}\|y\|} \\
& \times \exp \left\{-\frac{\sqrt{w^{2}+4}}{2 \varepsilon}\|y\|+\frac{1}{2 \varepsilon} w \cdot y\right\} .
\end{aligned}
$$

In our case:

$$
F(x)=\frac{m J}{\|x\|^{2}+a^{2}} \exp (-d(x)) .
$$

Suppose now that $x_{0}$ is point of the boundary of plasma, $x_{0} \in \partial \Omega$ and let in an $\mathcal{O}\left(\varepsilon^{p}\right)$ ball $U$ around $x_{0}$ with some $p<1$, the plasma be contained in a half space $H$ with outward normal $\nu$ and $x_{0} \in \partial H$, i.e.

$U \cap \Omega \subset\left\{x \mid\left(x-x_{0}\right) \cdot \nu \leq 0\right\}$.

If in $U \cap \Omega$ the function $F$ is continuous and almost constant, i.e.

$F(x)=F\left(x_{0}\right)+o(1)$ for $x \in U \cap \Omega$,

then one easily derives from (3.1.2) that

$1 \leq F\left(x_{0}\right) \frac{1}{2}\left(1+\frac{w \cdot \nu\left(x_{0}\right)}{\sqrt{\left(w \cdot \nu\left(x_{0}\right)\right)^{2}+4}}\right)+o(1)$.

The equality sign holds true if the plasma $U \cap \Omega$ contains a sphere $\left\{x \mid\left\|x-x_{0}-\varepsilon^{p} \nu\left(x_{0}\right)\right\| \leq \varepsilon^{p}\right\}$ with $p<1$. In the case where

$U \cap \Omega \supset U \cap\left\{x \mid x-x_{0} \cdot \nu\left(x_{0}\right) \leq 0\right\}$,

the inequality holds in reversed direction,

$1 \geq F\left(x_{0}\right) \frac{1}{2}\left(1+\frac{w \cdot \nu\left(x_{0}\right)}{\sqrt{\left(w \cdot \nu\left(x_{0}\right)\right)^{2}+4}}\right)+o(1)$.

An equality sign holds true in (3.1.8) if the nonplasma region $U \backslash \Omega$ contains a sphere

$\left\{x \mid\left\|x-x_{0}+\varepsilon^{p} \nu\left(x_{0}\right)\right\| \leq \varepsilon^{p}\right\} \quad$ with $p<1$.

Now we are ready to consider points where the free surface $S_{\varepsilon}$ becomes singular for $\varepsilon \downarrow 0$. Let us suppose that in some neighbourhood of $x_{0}$, two pieces $S_{0}^{\prime}, S_{0}^{\prime \prime}$ of the surface $S_{0}$ come together with an edge passing though $x_{0}$. Let $s$ denote the unit vector tangent to this edge at $x_{0}$. Taking the intersection of the free boundary $S_{0}$ with the plane $\Pi$ perpendicular to this edge, we notice that there are two possibilities to form an angle out of two pieces of surfaces satisfying (3.1). Indeed eq. (3.1) can be written as

$w_{\pi} \cdot n=2 \beta / \sqrt{1-\beta^{2}}$,

where $w_{\pi}$ is the projection of $w$ onto the plane $\Pi$, and since $n$ must be orthogonal to $s$ this equation gives us two possible solutions for $n$. For negative $\beta$ the situation is sketched in figs. $6 \mathrm{a}$ and fig. $6 \mathrm{~b}$. For positive $\beta$ it is shown in figs. $6 \mathrm{c}$ and fig. $6 \mathrm{~d}$.

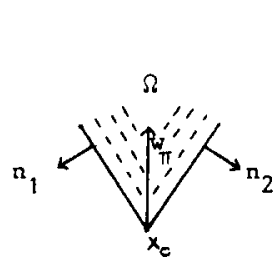

(a)

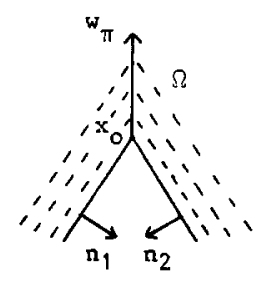

(b)

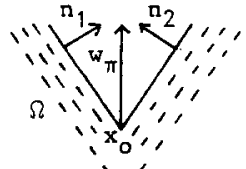

(c)

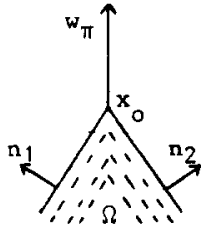

(d)

Fig. 6. Possible intersections of the free boundary for negative ((a) and (b)) and positive ((c) and (d)) values of $\beta$. 


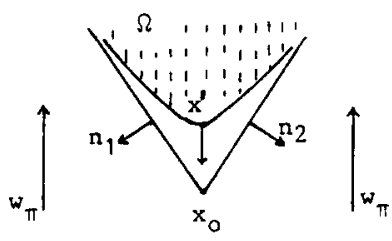

Fig. 7.

We shall now show that cases a) and c) lead to contradictions. Suppose that $\varepsilon>0$, but small enough. In such a case the free boundary is smooth in the vicinity of $x_{0}$ and approaches $x_{0}$ for $\varepsilon \downarrow 0$, fig. 7. Let $x^{\prime}$ will be a point on the plane $\Pi$ where $w_{\pi}$ enters plasma perpendicular to the intersection of its boundary with $\Pi$.

The difference between the outward normal to the plasma and $-w_{\pi} /\left\|w_{\pi}\right\|$ at $x^{\prime}$ is small, $o(1)$. Also the distance $d\left(x_{0}, x^{\prime}\right)$ is $o(1)$. In the case as shown on fig. 7 the intersection of the region occupied by plasma with the plane $\Pi$ is convex and since the curvature of the edge is of order $\mathcal{O}(\varepsilon)$ there exists a ball of radius $\mathcal{O}(1)$ which is tangent to the boundary of plasma at $x^{\prime}$ and which lies outside of $\Omega$. Clearly, we have then

$$
\begin{aligned}
& w_{\pi}\left(x^{\prime}\right) n\left(x^{\prime}\right)=-\left\|w_{\pi}\left(x^{\prime}\right)\right\|+o(1) \\
& =-\left\|w_{\pi}\left(x_{0}\right)\right\|+o(1), \\
& F\left(x^{\prime}\right)=F\left(x_{0}\right)+o(1) \text {. }
\end{aligned}
$$

Applying the formula (3.1.6) to the point $x^{\prime}$ we obtain

$$
1 \leq \frac{1}{2} F\left(x_{0}\right)\left(1-\frac{\left\|w_{\pi}\right\|}{\sqrt{\left\|w_{\pi}\right\|^{2}+4}}\right)+o(1) .
$$

On the other hand on the solutions of the Hamilton-Jacobi equation passing through $x_{0}$ with normals $n_{1}$ and $n_{2}$ the following equality:

$1=\frac{1}{2} F\left(x_{0}\right)\left(1+\frac{w_{\pi} \cdot n_{i}}{\sqrt{\left(w_{\pi} \cdot n_{i}\right)^{2}+4}}\right), \quad i=1,2$,

is satisfied which is just another form of eq. (2.2).
Since, however there is an $\mathcal{O}(1)$ difference between $n$ and $n_{1}$ or $n_{2}$, in fact $n=(1 / \sqrt{2})\left(n_{1}+n_{2}\right) /(1+$ $\left.n_{1} \cdot n_{2}\right)$, we arrive at a contradiction. This does not occur in cases as shown in fig. 6b. Also the application of the inequality (3.1.8) does not lead to any contradiction. On the other hand applying (3.1.8) to the case c) we are led again to a contradiction. This does not happen however for corners of the type d).

Summarizing: the types a) and c) of corners cannot be obtained asymptotically for $\varepsilon \downarrow 0$. We call them inadmissible corners. The other types of corners are called admissible and as a matter of fact we can show that solutions with this type of limit of the free surface do exist. The same reasoning applies to cases of corners made out of pieces of surface satisfying (2.8).

\subsection{Two-dimensional case}

In previous sections we analysed the possible local features of an asymptotic solution, such as layers, the matching of the rigid boundary with the $\mathrm{H}-\mathrm{J}$ surface and appearance of corners. Having all these necessary elements to our disposal we will try to put them together in order to construct a global approximation of the solution. In this section we consider the intersection of the globally defined free surface with the plane of symmetry.

Since the characteristics of the Hamilton-Jacobi equations stay in the plane of symmetry this is more or less a two-dimensional problem. Let us first consider the intersection curve of this plane of symmetry with the right free surface. We shall distinguish between various cases dependent of the wind direction. We denote the opening angle of the cone by $\theta_{0}$. For $\alpha$, the angle of the wind vector with the negative central axis of the cone, we shall distinguish between several cases as indicated in fig. 8 .

The points where the curve meets the lower halfline $\theta=-\theta_{0}$ and the upper halfline $\theta=\theta_{0}$ are denoted by $x_{1}$ and $x_{\mathbf{u}}$, respectively. In fig. 4 it was shown that $r_{1}=\left\|x_{1}\right\| \geq \rho_{-}$and $r_{\mathrm{u}}=\left\|x_{\mathrm{u}}\right\| \geq \rho_{-}$. In order to have consistency for the transition of the 


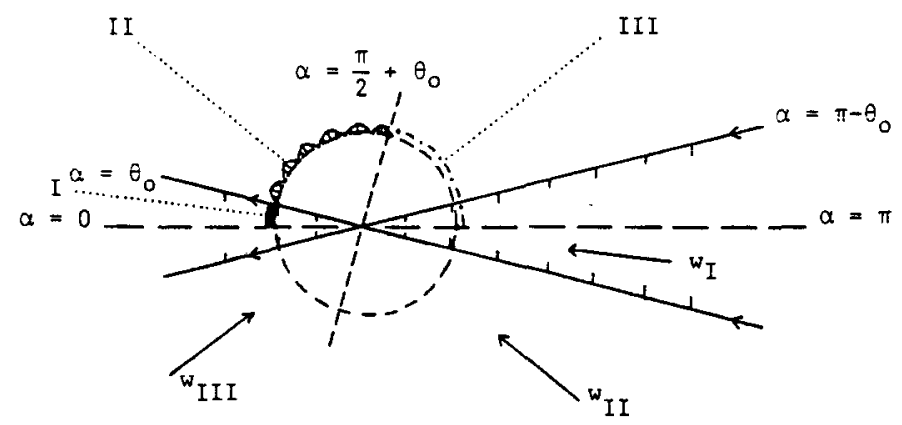

Fig. 8.

free surface to the rigid part of the boundary of the cone it is necessary that (2.6) is satisfied. This yields the following a priori information (see fig. 9):

$\rho_{-} \leq r_{1} \leq \rho_{1}, \quad \rho_{-} \leq r_{\mathrm{u}} \leq \rho_{\mathrm{u}}$,

with

$\rho_{1, \mathrm{u}}=\sqrt{\frac{1}{2} m\left(1+\beta_{1, \mathrm{u}}\right)-a^{2}}$,

$\beta_{1, \mathrm{u}}=\frac{w \cdot \nu\left(r_{1, \mathrm{u}}\right)}{\sqrt{4+\left(w \cdot \nu\left(r_{1, \mathrm{u}}\right)\right)^{2}}}$,

$w \cdot \nu\left(r_{1}\right)=\|w\|\left\{\cos \alpha \sin \theta_{0}-\sin \alpha \cos \theta_{0}\right\}$,

$w \cdot \nu\left(r_{\mathrm{u}}\right)=\|w\|\left\{\cos \alpha \sin \theta_{0}+\sin \alpha \cos \theta_{0}\right\}$.

Let us start with a discussion of the case

$\mathrm{I}: 0 \leq \alpha \leq \theta_{0}$

It is clear that there has to be a point $P$ on the curve where the normal equals $-w /\|w\|$. Using eq. (3.1) it follows that at this point $\mu=-1$ while the free surface has to lie completely in the region $\mu \geq-1$. Consequently, the coordinates of $P$ have to be

$x_{P}=-\frac{w}{\|w\|} \rho_{-}$.

From the point of view of position $x_{P}$ plays the role of a stationary point.

Another way to look for the curve is by a parametrization

$r=R(\theta)$.

Substitution of the expression for the normal into (3.2) provides us with the following O.D.E.:

$\frac{1}{R} \frac{\mathrm{d} R}{\mathrm{~d} \theta}=\left\{-w_{r} w_{\theta} \pm \mu \sqrt{1-\mu^{2}}\right\} /\left(\mu^{2}-w_{\theta}^{2}\right)$,

where $w_{r}, w_{\theta}$ denote the components of $w /\|w\|$ in

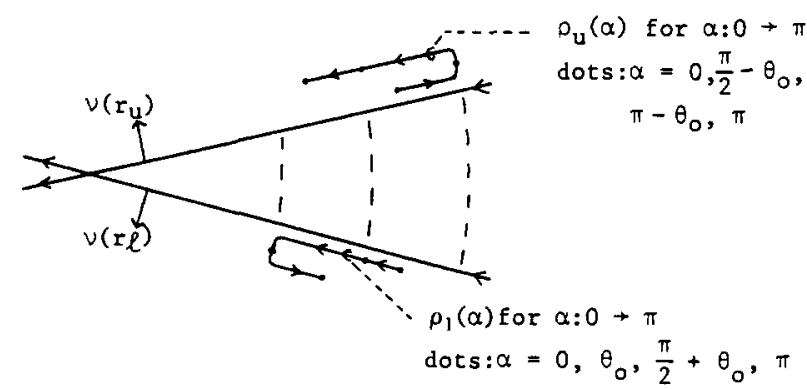

Fig. 9. $\rho_{\mathrm{u}}(\alpha)$ and $\rho_{1}(\alpha)$ are the last possible positions for the free surface to separate from the upper- and lower part of the cone, respectively. They vary with the wind angle $\alpha$, as shown here. 


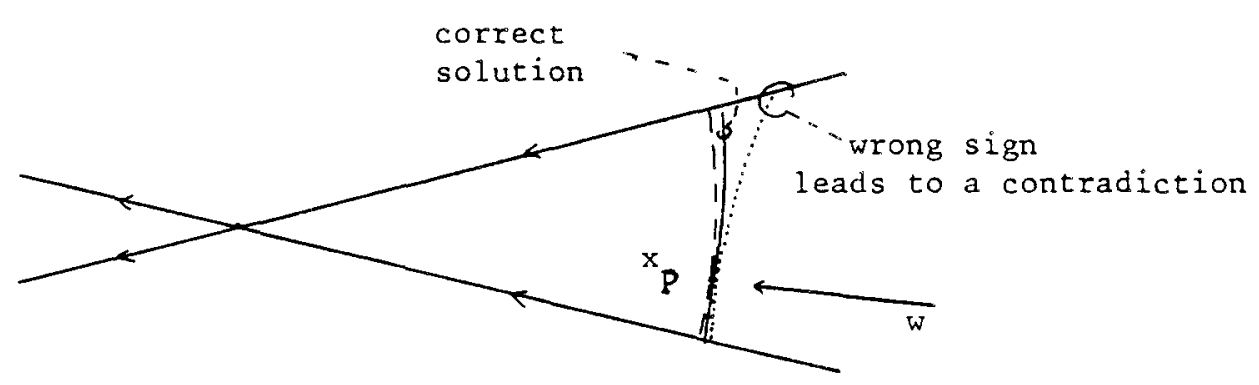

Fig. 10

the directions $e_{r}=(\cos \theta, 0, \sin \theta)$ and $e_{\theta}=$ $(-\sin \theta, 0, \cos \theta)$, respectively, i.e.

$$
\begin{aligned}
w_{r}(\theta) & =-\cos \alpha \cos \theta+\sin \alpha \sin \theta \\
& =-\cos (\theta+\alpha), \\
w_{\theta}(\theta) & =\cos \alpha \sin \theta+\sin \alpha \cos \theta \\
& =\sin (\theta+\alpha) .
\end{aligned}
$$

One of the central issues in an equation like (3.2.4) is to decide about the sign. Here the correct choice will be the + sign for $\theta>-\alpha$ and the - sign for $\theta<-\alpha$. Let us first discuss why, once these signs are chosen in this way, there is an acceptable non-trivial solution starting from the critical point.

The point $\theta=-\alpha, R=\rho_{-}$is a stationary point of (3.2.4) where the equation is non-Lipschitzian. Nevertheless, there is a unique solution with the following behaviour:

$$
R(\theta)=\rho_{-}+\frac{1}{2} C(\theta+\alpha)^{2}+\cdots .
$$

Substitution in (3.2.4) yields the following nontrivial value for $C, C=\rho_{-} /\left(1+\rho_{-} \sqrt{\mu^{\prime}\left(\rho_{-}\right)}\right)$. Thus the curve starting from the critical point $\left(-\alpha, \rho_{-}\right)$ has been determined. However, it is necessary to check whether the points where this curve reaches the boundary of the cone are in agreement with condition (3.2.1) and whether a correct transition to the rigid part of the plasma front is possible. As for the transition to the rigid part of the surface at a point satisfying (3.2.1) we are in the situation discussed in the context of (2.8), i.e. a transition on an $\mathcal{O}(b)$ scale consisting of one branch or of two branches making a corner of admissible type.
However, if we would have chosen the other sign in (3.2.4) a contradiction would arise in this transition. Either there would be a conflict with monotonicity in the case of one branch or the corner would be of inadmissible type in the case of two branches. This forces us to choose the signs in the way we did (see fig. 10).

From the field of directions corresponding to (3.2.4) it is clear that $\rho_{-}<R(\theta)<\rho_{0}$ for $\theta<-\alpha$. Hence

(i) $R\left(-\theta_{0}\right)<\rho_{1}(\alpha), \quad$ (see fig. 9 ).

Analogously, if $\alpha<\pi / 2-\theta_{0}$ one also has $\rho_{-}<$ $R(\theta)<\rho_{0}$ for $\theta>-\alpha$. Therefore

(ii) $R\left(\theta_{0}\right)<\rho_{u}$ for $\alpha<\frac{\pi}{2}-\theta_{0}$.

Note that the condition $\alpha<\pi / 2-\theta_{0}$ is satisfied if $\theta_{0}<\pi / 4$. For large openings angles $\theta_{0}>\pi / 4$ and $\alpha>\pi / 2-\theta_{0}$ it cannot be excluded that $R\left(\theta_{0}\right)>$ $\rho_{\mathrm{u}}(\alpha)$. In that case the curve $R(\theta)$ cannot be valid all the way up to $\theta=\theta_{0}$. For some point $\theta_{1}$ with $\pi / 2-\alpha<\theta_{1}<\theta_{0}$ with $R\left(\theta_{1}\right)>\rho_{0}$ the free surface will turn around making a corner with two dimensional $\varepsilon \times \varepsilon$ layer behavior in the solution. (See fig. 11.) For the curve $r=R_{1}(\theta)$ there is only one acceptable possibility: it starts at the upper part of the boundary of the cone with

$R_{1}\left(\theta_{0}\right)=\rho_{\mathrm{u}}$.

This implies that the curve $r=R_{1}(\theta)$ is tangent to the cone at point $\left(\theta_{0}, \rho_{\mathrm{u}}\right)$. Further $R_{1}$ satisfies the 


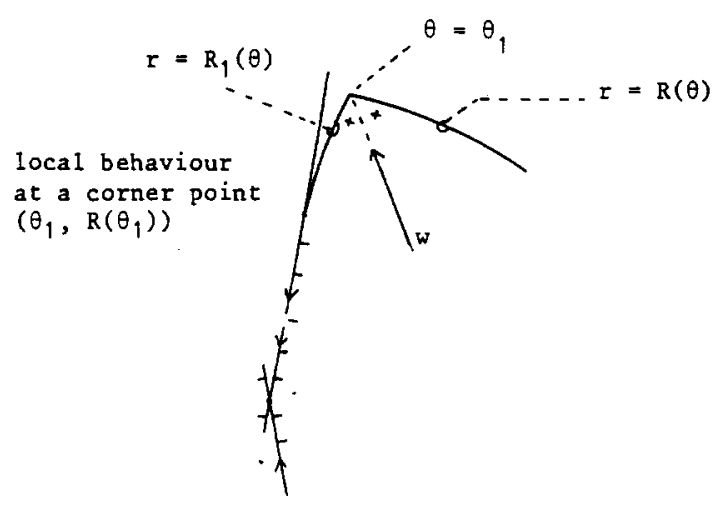

Fig. 11.

differential equation

$\frac{1}{R_{1}} \frac{\mathrm{d} R_{1}}{\mathrm{~d} \theta}=\left\{-w_{r} w_{\theta}-\mu \sqrt{1-\mu^{2}}\right\} /\left(\mu^{2}-w_{\theta}^{2}\right)$.

Note that (3.2.10) has a singularity for $\theta \rightarrow \theta_{0}$ and $R_{1} \rightarrow \rho_{\mathrm{u}}$. There is nevertheless a unique solution behaving as

$R_{1}(\theta)=\rho_{\mathrm{u}}+C \sqrt{\theta_{0}-\theta}+\mathcal{O}\left(\theta_{0}-\theta\right)$.

Substitution in (3.2.10) yields

$C=\sqrt{2 w_{\mathbf{r}}\left(\theta_{0}\right) / \mu^{\prime}\left(\rho_{-}\right)}$.

An alternative approach is to use (3.5) instead of (3.2.10) with the initial condition $x_{0}$ corresponding to (3.2.9) and $p_{0}=\nu\left(\theta_{0}\right)$ the normal to cone. The advantage is that in this way one only has to deal with an equation without singularities. $R_{1}(\theta)$ is uniquely determined for $\theta \leq \theta_{0}$ and it represents the free surface as long as $R_{1}(\theta) \leq R(\theta)$. Note, that the corner at $\left(\theta_{1}, R\left(\theta_{1}\right)\right)$ is consistent with the inequality in (3.1.6) for $\phi_{1}=\phi_{2}$ with $\phi_{1}$ and $\phi_{2}$ as in fig. 10. The equality $\phi_{1}=\phi_{2}$ follows from (3.1) and (3.2.4), (3.2.10).

II: $\theta_{0}<\alpha<\theta_{0}+\pi / 2$

Note that for $\alpha=\theta_{0}$ the critical point $x_{P}$ lies on the lower part of the boundary of the cone. As a

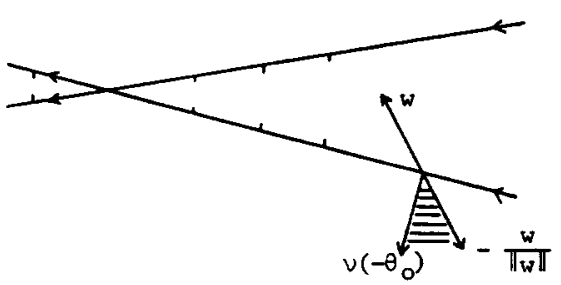

Fig. 12.

consequence we need for $\alpha>\theta_{0}$ a different kind of initial condition for the hamiltonian system (3.5). Consider a small neighbourhood of the point $\left(-\theta_{0}, R\left(\theta_{0}\right)\right)$ where the free surface leaves the lower part of the boundary of the cone as in fig. 3 . As a consequence of the argument on monotonicity a transition on an $\mathcal{O}(b)$ scale with one branch is possible, if the normal $n\left(-\theta_{0}\right)$ which serves as an initial condition $p_{0}$ for the Hamiltonian system (3.5) lies in the shaded sector in fig. 12. Let us now consider the solution globally.

The normal to the free surface cannot be equal to $-w /\|w\|$ at a value $r>R\left(\theta_{0}\right)$, for $\mu=-1$ occurs for the smallest possible value of $r$, see fig. 4. Therefore, the only acceptable way to begin is

$$
\begin{aligned}
& n\left(-\theta_{0}\right)=-\frac{w}{\|w\|}=p_{0} \\
& R\left(-\theta_{0}\right)=\rho_{-} .
\end{aligned}
$$

The curve can now be uniquely determined by solving (3.5) or by using a parametrization $r=$ $R(\theta)$ as in (3.2.4) with the + sign. The - sign leads to a contradiction analogous to before (see fig. 13).

If the point where this curve reaches the upper part of the boundary of the cone is such that

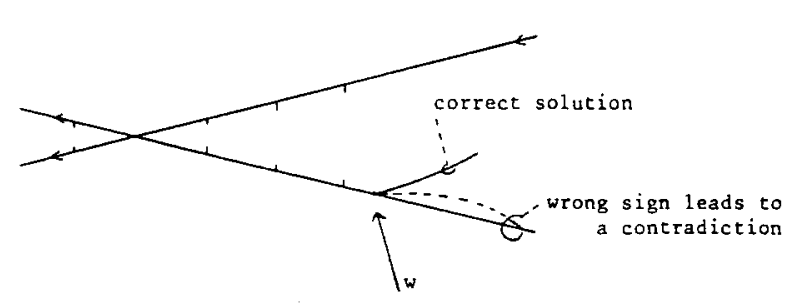

Fig. 13. 
$R\left(\theta_{0}\right)>\rho_{\mathrm{u}}$ then the free surface consists only partly of $r=R(\theta)$. Completely analogous to the previous case one needs another curve $r=R_{1}(\theta)$ starting at $\left(\theta_{0}, \rho_{\mathrm{u}}\right)$ tangently to the boundary of the cone. This second part of the curve can again be determined either by solving (3.5) or (3.2.10).

\section{III: $\pi / 2+\theta_{0}<\alpha<\pi$}

If $\alpha=\pi / 2+\theta_{0}$ the vector $-w /\|w\|$ coincides with $\nu\left(-\theta_{0}\right)$, the normal on the boundary of the cone, and for $\alpha>\pi / 2+\theta_{0}$ the start condition of the previous case does not make sense, since it is in conflict with starting from the lower boundary and staying inside the cone.

Reasoning on the basis of (3.1.6) as in the previous case we see that the only possibility is

$n\left(-\theta_{0}\right)=\nu\left(-\theta_{0}\right)$,

$R\left(-\theta_{0}\right)=\rho_{1}$,

i.e. the free surface starts tangently at the lower part of the cone and these are the start values for (3.5). In the representation $r=R(\theta)$ where $R$ satisfies (3.2.4) with a + sign one starts at a singular point of the equation as

$R(\theta)=\rho_{1}+C \sqrt{\theta+\theta_{0}}+\mathcal{O}\left(\theta+\theta_{0}\right)$,

where $C$ has the value $\sqrt{2 w_{r} / \mu^{\prime}\left(\rho_{l}\right)}$. Further, as for the necessity of a second part of the free surface starting tangently from the upper part of the boundary of the cone the situation is as before.

Summarizing, we conclude that the right free surface intersected with the plane of symmetry consists (i) either of 1 part valid from $\partial G_{1}$, the lower half-line of the boundary of the cone to $\partial G_{\mathrm{u}}$, the upper half-line (ii) or of 2 parts, one valid from $\partial G_{1}$ up to a corner point and one valid from this corner point to $\partial G_{\mathrm{u}}$. In situation (i) or in situation (ii) for the lower part of the curve there are three start possibilities:

I: from a critical point $P$ for $0 \leq \alpha \leq \theta_{0}$;

II: from $P \in \partial G_{1}$ with a direction perpendicular to the wind for $\theta_{0}<\alpha \leq \theta_{0}+\pi / 2$; and

III: from $P \in \partial G_{1}$ starting tangently to $\partial G_{1}$ for $\theta_{0}+\pi / 2<\alpha<\pi$.

The second situation arises for $\alpha_{0}<\alpha<\pi$ with $\pi / 2-\theta_{0}<\alpha_{0}<\pi-\theta_{0}$. The first bound for $\alpha_{0}$ follows from (3.2.7). The second bound comes from the observation that in case II and III the lower part of the curve is at the right of the line through $P$ with the direction of the wind as long as $r \leq \rho_{0}$. In combination with the behaviour of $\rho_{\mathrm{u}}$ shown in fig. 5 it is clear that then $R\left(\theta_{0}\right)>\rho_{\mathrm{u}}$. The upper part of the curve starts from $P \in \partial G_{\mathrm{u}}$ tangently to $\partial G_{\mathrm{u}}$ and turns backwards till it meets the lower part.

The construction of the intersection of the left part of the free surface with the plane of symmetry proceeds in an analogous way, but there are some interesting geometrical difference stemming from the shape of the right part of the free surface.

We shall give a description of various situations occurring as $\alpha$ runs down from $\pi$ to 0 . For $\alpha$ equal to or close to $\pi$ the situation will be as in fig. 14.

Our by now familiar reasoning leads us to a left curve consisting of two parts $r=-\tilde{R}(\theta)<0$ and $r=-\tilde{R}_{1}(\theta)<0$ meeting at an obtuse angle at

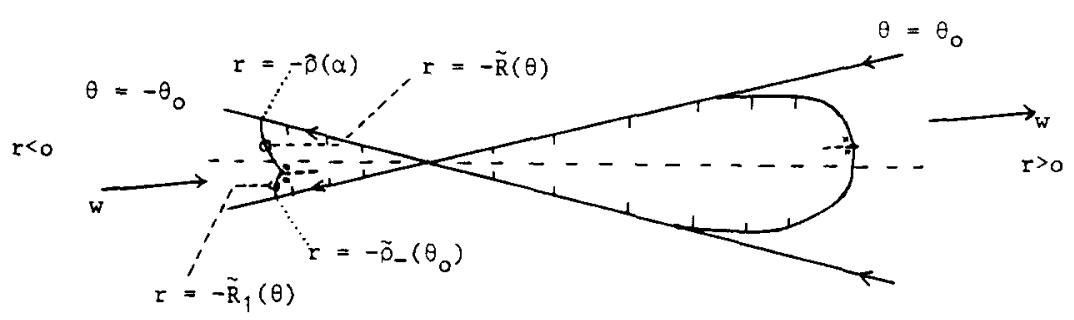

Fig. 14. 
$\theta=\tilde{\theta_{1}}$ satisfying

$$
\begin{aligned}
& \frac{1}{\tilde{R}} \frac{\mathrm{d} \tilde{R}}{\mathrm{~d} \theta}=\left\{-w_{r} w_{\theta}+\tilde{\mu} \sqrt{1-\tilde{\mu}^{2}}\right\} /\left(\tilde{\mu}^{2}-w_{\theta}^{2}\right), \\
& \tilde{R}\left(-\theta_{0}\right)=\hat{\rho}(\alpha) ;
\end{aligned}
$$

$\frac{1}{\tilde{R}} \frac{\mathrm{d} \tilde{R}_{1}}{\mathrm{~d} \theta}=\left\{-w_{r} w_{\theta}-\tilde{\mu} \sqrt{1-\tilde{\mu}^{2}}\right\} /\left(\tilde{\mu}^{2}-w_{\theta}^{2}\right)$,

$\tilde{R}_{1}\left(\theta_{0}\right)=\tilde{\rho}_{-}\left(\theta_{0}\right)$,

with $w_{r}$ and $w_{\theta}$ as in (3.2.6). The lower part of the curve starts perpendicular to the wind at the boundary of the cone. The start of the upper part of the curve is at the value $\hat{\rho}(\alpha)$. Here $\hat{\rho}(\alpha)$ is a certain constant which will follow by the analysis of the full three-dimensional case further on. For $\alpha=\pi$ we have of course $\hat{\rho}(\pi)=\tilde{\rho}_{-}\left(-\theta_{0}\right)$. It is somewhat surprising that even in the plane of symmetry the three-dimensionality of the problem plays a role at first order, but this does not lead to any contradictions. In the purely two-dimensional problem considering from the onset only the $x$ and $z$ direction the start position would be characterized just by $\tilde{\rho}_{-}\left(-\theta_{0}\right)$ for $\alpha$ sufficiently close to $\pi$. The corner at $\tilde{\theta}_{1}$ is one of an admissible type, see (3.1.8).

When $\alpha$ decreases the corner point at $\theta=\tilde{\theta}_{1}$ will move to the position $\theta=-\theta_{0}$, where it arrives for $\alpha=\alpha_{1}$. For $\alpha<\alpha_{1}$ and $\alpha$ sufficiently close to $\alpha_{1}$ the left free surface consists only of the curve $r=-\tilde{R}_{1}(\theta)$ satisfying (3.2.14). With a further decrease of $\alpha$ two things occur:

a) if $-\tilde{R}_{1}\left(\theta_{0}\right)<-\tilde{\rho}_{\mathrm{u}}\left(-\theta_{0}\right)$ then the left free surface has a second part $r=-\tilde{R}_{2}(\theta)$ starting tangently to the cone at $\theta=-\theta_{0}, r=-\tilde{\rho}_{\mathrm{u}}\left(-\theta_{0}\right)$ and satisfying (3.5) or the equation

$\frac{1}{\tilde{R}_{2}} \frac{\mathrm{d} \tilde{R}_{2}}{\mathrm{~d} \theta}=\left\{-w_{r} w_{\theta}+\tilde{\mu} \sqrt{1-\tilde{\mu}^{2}}\right\} /\left(\tilde{\mu}^{2}-w_{\theta}^{2}\right)$,

b) if $\alpha<\pi / 2-\theta_{0}$, the start point of $r=-\tilde{R}_{1}\left(\theta_{0}\right)$ changes to

$-\tilde{R}_{1}\left(\theta_{0}\right)=-\tilde{\rho}_{1}\left(\theta_{0}\right)$.

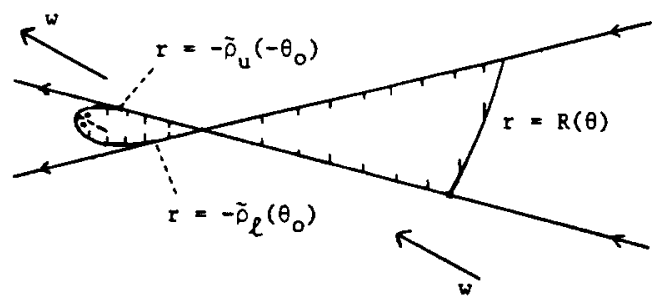

Fig. 15.

The interpretation of $-\tilde{\rho}_{\mathrm{u}}\left(-\theta_{0}\right)$ and $-\tilde{\rho}_{1}\left(\theta_{0}\right)$ is the same $\rho_{\mathrm{u}}$ and $\rho_{1}$ in (3.2.1). They are implicitly given by

$$
\begin{aligned}
& \frac{w}{\|w\|} \cdot \tilde{\nu}\left(-\theta_{0}\right)=\tilde{\mu} \quad \text { at } r=-\tilde{\rho}_{\mathrm{u}}\left(-\theta_{0}\right), \\
& \frac{w}{\|w\|} \cdot \tilde{\nu}\left(\theta_{0}\right)=\tilde{\mu} \quad \text { at } r=-\tilde{\rho}_{\mathrm{u}}\left(\theta_{0}\right),
\end{aligned}
$$

with $\tilde{\nu}$ the outward normal on the boundary of the cone, compare (2.9-10). Herewith the picture of the situation in the plane of symmetry is complete, apart from $\hat{\rho}(\alpha)$. In fig. 15 we show another representative case.

Of course, in the special cases $\alpha=0$ and $\alpha=\pi$ the three-dimensional picture of the plasma has now been determined, because of rotational symmetry along the central axis. The three-dimensional picture in other cases is discussed in the next section.

\subsection{On the free surface lying in three- dimensional space}

Here we shall extend the results of the previous section for the curve in the plane of symmetry to obtain a surface which makes a full cross section of the cone. To begin we will discuss the right part of the free surface. We remind the reader that in terms of the angles $\theta$ and $\phi$ introduced in section 2 the boundary of the cone is given by

$\partial G=\left\{\sin ^{2} \theta+\cos ^{2} \theta \cdot \sin ^{2} \phi=\sin ^{2} \theta_{0}\right\}$,

where the angles are such that $x=r \cos \theta \cos \phi$, $y=r \cos \theta \sin \phi$ and $z=r \sin \theta$. Sometimes it will 
be convenient to work with the angle $\chi \in[-\pi, \pi]$, the argument of $(y,-z)$ in the $(y, z)$ plane, i.e. $y=s \sin \chi, z=-s \cos \chi$, with $s=\sqrt{y^{2}+z^{2}}$. Note, that $\chi=0, \pi$ corresponds to $\phi=0, \theta<0, \theta>0$, respectively.

Let us denote by $r=L(\chi)$ the curve where the right free surface intersects $\partial G$, i.e. $L(0)=r_{1}$, $L(\pi)=r_{\mathrm{u}}$, see (3.2.1). Analogously to (3.2.1) we now have a priori information of the form

$\rho_{-} \leq L(\chi) \leq \rho(\chi)$.

Here $\rho(\chi)$ is the largest position for which a consistent transition of the free surface to the boundary of the cone can be made, i.e. (see (2.6))

$$
\begin{aligned}
& \frac{w}{\|w\|} \cdot \nu(\chi)=\mu(\rho(\chi)), \\
& \rho(\chi)=\sqrt{\frac{m}{2}\left\{1+\frac{w \cdot \nu(\chi)}{\sqrt{4+(w \cdot \nu(\chi))^{2}}}-a^{2}\right\}},
\end{aligned}
$$

with $\nu(\chi)$ the outward normal on the boundary of cone and

$w \cdot \nu(\chi)=\|w\|\left\{\cos \alpha \sin \theta_{0}-\sin \alpha \cos \theta_{0} \cos \chi\right\}$.

Let us analyse the inner product in (3.3.3). If $0 \leq \alpha<\theta_{0}$ then $w \cdot \nu(\chi)$ is always positive the wind blows in an outward direction through all of $\partial G_{+}=$right part of $\partial G$. If $\pi-\theta_{0}<\alpha \leq \pi$ then $w \cdot \nu(\chi)$ is always negative and the wind blows inwards on all of $\partial G_{+}$. If $\theta_{0}<\alpha<\pi-\theta_{0}$ then there exists an angle $\chi_{1}$ such that $w \cdot \nu\left(\chi_{1}\right)=0$. This angle is given by

$\cos \chi_{1}=\operatorname{tg} \theta_{0} / \operatorname{tg} \alpha$.

For angles $\chi$ with $|\chi|<\left|\chi_{1}\right|$ the wind blows inwards with respect to $\partial G_{+}$and for angles $\chi$ with $|\chi|>\left|\chi_{1}\right|$ the wind blows outwards with respect to $\partial G_{+}$. At the special values $|\chi|=\left|\chi_{1}\right|$ the wind is tangent to $\partial G_{+}$.
Further we observe that $w \cdot \nu(\chi)$ and $\rho(\chi)$ are both increasing functions of $|\chi|$ and $\rho_{\mathrm{e}}=\rho(0) \leq$ $\rho(\chi) \leq \rho(\pi)=\rho_{\mathrm{u}}$. We will now take a closer look at cases I, II, III as in the previous section.

\section{$\mathrm{I}: 0 \leq \alpha \leq \theta_{0}$}

The complete free surface can be found by sending characteristics from the critical point. Take a small circle with the radius $r_{0}>0$ in the plane perpendicular to $w$ through the critical point $x_{p}$ (where the wind is perpendicular to the surface $\mu=-1$, compare (3.3.1)) as the initial curve $x_{0}(s)$ in (3.5). As initial values for $p$ one can take $p_{0}(s)=x_{0}(s)-\alpha w /\|w\|$, where in order to satisfy (3.6) $\alpha$ has to be such that $\rho_{-}+\alpha=r_{0}|\mu| / \sqrt{1-\mu^{2}}$ with $\mu=\mu\left(\sqrt{\rho_{-}^{2}+r_{0}^{2}}\right)$. In this way $(3.7)_{\mathrm{nch}}$ is satisfied and characteristics run in all directions forming a surface that crosses the cone. The surface we are looking for, is found in the limit $r_{0} \downarrow 0$. Since $H$ is not Lipschitzian for $\mu=-1$ we obtain however similarly to the two dimensional case, two surfaces which are tangent at $x_{P}$. The first leads to a plasma which is locally convex in the vicinity of $x_{P}$. The other one which leads to concave plasma must be rejected for global reasons i.e. there is no consistent matching with the rigid part of boundary.

Let us denote the proper one by $r=R(\theta, \phi)$. Of course $r=R(\theta, 0)$ coincides with the curve constructed in (3.2.4-6). Again we have to check (3.3.1). Because of (3.10a) this surface lies in the half-space where $w /\|w\| \geq-\rho_{-}$. From the field of directions corresponding to the Hamiltonian system it is clear that a characteristic cannot leave the region $r \leq \rho_{0}$ at a point $\rho_{0}, \theta, \phi$, where

$$
\left.\frac{\mathrm{d}}{\mathrm{d} t}\left\{\frac{1}{2}\|x\|^{2}\right\}\right|_{r=\rho_{0}}=w \cdot x /\|w\|<0
$$

Hence, a characteristic can only cross the sphere $r=\rho_{0}$ in the half-space $w \cdot x>0$.

In combination with the behaviour of $\rho(\chi)$ in (3.3.2) this yields that if $\alpha \leq \pi / 2-\theta_{0}$ the curve where the free surface meets $\partial G_{+}$satisfies (3.3.1). 
If $\alpha>\pi / 2-\theta_{0}$ there is the possibility

$R(\theta, \phi) \mid \partial_{G_{+}}>\rho(\chi)$ in the region $|\chi| \geq\left|\chi_{2}\right|$

with

$\cos \chi_{2}=-\operatorname{tg} \theta_{0} / \operatorname{tg} \alpha$.

Analogous to section 3.2.I, we must then calculate the surface $r=R_{1}(\theta, \phi)$ which we started tangently to the boundary of the cone along $r=\rho(\chi)$ for $\chi_{2} \leq \chi \leq \pi$. Therefore we solve (3.5) with the initial conditions

$x_{0}(\chi)=\rho(\chi) e(\chi)$

with

$$
\begin{aligned}
e(\chi) & =\left(\cos \theta_{0}, \sin \theta_{0} \sin \chi,-\sin \theta_{0} \cos \chi\right) \\
p_{0}(\chi) & =\nu(\chi) \\
& \text { def } \\
& =\left(-\sin \theta_{0},-\cos \theta_{0} \sin \chi,-\cos \theta_{0} \sin \chi\right)
\end{aligned}
$$

while $t$ runs backwards. The free surface at $\theta, \phi$ is found as the minimum of $R(\theta, \phi)$ and $R_{1}(\theta, \phi)$.

\section{II: $\theta_{0}<\alpha \leq \theta_{0}+\pi / 2$}

In section 3.2.II we obtained as starting values on the lower part of $\partial G_{+}$for the characteristic with $\phi=0$

$\chi=0, \quad x_{0}(0)=\rho_{-} e(0), \quad p_{0}(0)=-\frac{w}{\|w\|}$,

with $e(\chi)$ as in (3.3.5). It is reasonable to expect that in order to construct the complete surface $r=R(\theta, \phi)$ we need a start curve

$x_{0}(\chi)=L(\chi) \cdot e(\chi)$ for $0 \leq|\chi| \leq\left|\chi_{1}\right|$,

that is an initial curve at the part of $\partial G_{+}$where the wind enters the cone. This curve can be determined by using the monotonicity property of eq. (2.8). The separation of the layer takes place in such a way that $w \cdot n$ has to decrease as a function of $\xi$. Let $x_{0}$ be a point of the separation curve and let $\Pi$ be a plane perpendicular to the curve at $x_{0}$. If $n$ is the outward normal to the $\mathrm{H}-\mathrm{J}$ surface at $x_{0}$ then we have

$w \cdot n=w_{\pi} \cdot n$,

where $w_{\pi}$ is the projection of $w$ on the plane $\Pi$, which is given by

$w_{\pi}=w-\left(w \cdot \frac{\tau}{\|\tau\|}\right) \cdot \frac{\tau}{\|\tau\|}$,

.with a tangent vector

$\tau=\left(\mathrm{d} L\left(\chi_{0}\right) / \mathrm{d} \chi\right) \cdot e_{r}+L \sin \theta_{0} \cdot e_{\chi}$.

The unit vectors $e_{r}, e_{\chi}$ are lying in the tangent plane to the cone in radial and $\chi$-angular direction, respectively, i.e. $e_{r}=e(\chi)$ as in (3.3.5) and $e_{\chi}=(0, \cos \chi, \sin \chi)$. According to our discussion concerning eq. (2.8), the separation can only occur at places where $w \cdot n$ decreases when passing from the rigid branch to the $\mathrm{H}-\mathrm{J}$ branch of the free surface, but more can be said. Analogous to the two-dimensional case the transition can take place only when $w_{\pi} \cdot n$ assumes its minimal value (i.e. $\left|w_{\pi} \cdot n\right|$ attains it maximal value) otherwise there is no possibility to construct the global surface. Indeed if this was the case then the characteristics starting from the separation curve would drift in direction opposite to the wind, thus never reaching the upper part of the cone, compare fig. 13. This provides us with the following equation:

$-w_{\pi} /\left\|w_{\pi}\right\|=n\left(x_{0}\right)$.

Using the Hamilton-Jacobi equation we obtain from (3.3.8) the following results:

$$
\begin{aligned}
& -\left.\frac{\left\|w_{\pi}\right\|}{\|w\|}\right|_{x=L(\chi) e(x)}=\mu(L(\chi)), \\
& (w \cdot \tau)^{2}=\|\tau\|^{2}\left(1-\mu^{2}\right) .
\end{aligned}
$$

The situation is complicated because (3.3.8-9) automatically imply, that $(3.7)_{\mathrm{ch}}$ holds, i.e. $x_{0}(\chi)$ is a semi-characteristic curve. However $w_{\pi}$ cannot be perpendicular to $\partial G_{+}$, unless we are at the maximal start position of the free surface $\rho(\chi)$. Therefore $w_{\pi} \cdot x_{0}(\chi) \neq 0$ and we can guarantee that (3.8) can be satisfied with $p_{0}(\chi)$ proportional to $w_{\pi}$. 
In a straightforward manner we derive from (3.3.9) a differential equation for $L(\chi)$

$$
\begin{aligned}
\frac{1}{L} \frac{\mathrm{d} L}{\mathrm{~d} \chi}= & \sin \theta_{0}\left\{\left[-w_{r} \cdot w_{\chi}\right.\right. \\
& \left. \pm \sqrt{\left(w_{r}^{2}+w_{\chi}^{2}+\mu^{2}-1\right)\left(1-\mu^{2}\right)}\right] \\
& \left./\left[w_{r}^{2}+\mu^{2}-1\right]\right\},
\end{aligned}
$$

with $\mu(L)$ as in (3.2) and $w_{r}, w_{\chi}$ the components of $w /\|w\|$ tangential to the cone in radial and $\chi$-angular direction, respectively

$$
\begin{aligned}
& w_{r}(\chi)=-\cos \alpha \cos \theta_{0}-\sin \alpha \sin \theta_{0} \cos \chi, \\
& w_{\chi}(\chi)=\sin \alpha \sin \chi .
\end{aligned}
$$

Since $L(\chi)=L(-\chi)$ we have only to determine the part of the curve with $\chi \geq 0$. In order to choose the correct sign in eq. (3.3.10) we consider the position of each of these curves with respect to the wind direction.

Let $\tau_{-}$and $\tau_{+}$denote the directions tangent to the curves defined by (3.3.10) with minus and plus sign, respectively. From fig. 16 one easily sees that when $\tau_{-}$is chosen as the vector tangent to the separation curve then the wind (or its projection) is leaving the plasma (which is supposed to be on

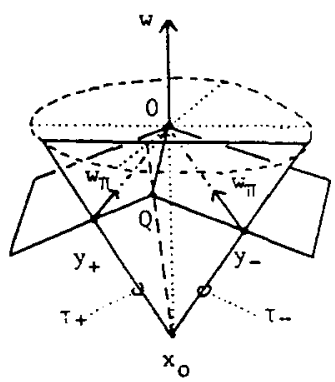

Fig. 16. The intersection of the cone defined by eq. (3.3.9b) with the plane tangent to the surface of the cone $G$. The focal point is on the left-hand side. $x_{0}, y_{+}, y_{-}$determine the plane tangent to $\partial G$ at $x_{.} ; 0 Q$ is perpendicular to this plane and $\left(x_{0}, y_{+}\right) \perp\left(y_{+}, Q\right),\left(x_{0}, y_{-}\right) \perp\left(y_{-}, Q\right) ;\left(y_{+}, 0\right)$ and $\left(y_{-}, 0\right)$ are in the direction of $w_{\pi}$ corresponding to $\tau_{+}$and $\tau_{--}$, respectively. the left side). Therefore (3.3.8) is violated unless we take plus sign in eq. (3.3.10).

Note that eq. (3.3.10) is defined on $\partial G_{+}$where $0 \leq \chi \leq \chi_{1}$ and $w_{r}^{2}+w_{\chi}^{2}+\mu^{2}-1 \geq 0$. The latter inequality is equivalent to $L(\chi) \leq \rho(\chi)$.

The initial condition has already been derived, see (3.3.6),

$$
L(0)=\rho_{-} .
$$

Again we need to deal with the complication that we start from a point where the equation is nonLipschitzian. There is a unique solution starting as

$L(\chi)=\rho_{-}+\frac{1}{2} C \chi^{2}+\mathcal{O}\left(\chi^{2}\right)$.

The constant $C>0$ is found by substitution in (3.3.10)

$w_{r}(0) C /\left(\rho_{-} \sin \theta_{0}\right)=-\sin \alpha-\sqrt{\mu^{\prime}\left(\rho_{-}\right) C}$.

For the curve $L(\chi)$ there are two possibilities: (i) it runs all the way to $\chi=\chi_{1}$, or (ii) it ends on the curve $r=\rho(\chi)$ before it reaches $\chi=\chi_{1}$. The first possibility occurs for $\theta_{0}<\alpha<\alpha_{c}$ and the second for $\alpha_{c}<\alpha<\theta_{0}+\pi / 2$. Fig. 17 shows the projection of the curves $r=L(\chi)$ and $r=\rho(\chi)$ on the $x-z$ plane in two representative cases (see fig. 17). Hence the initial conditions for the Hamiltonian system consist of the curve $x_{0}(\chi)=L(\chi) \cdot e(\chi)$ with $p_{0}(\chi)=-w_{\pi}(\chi)$ for $|\chi| \leq\left|\chi_{\mathrm{c}}\right|$ where $\chi_{\mathrm{c}}=$ $\max \left\{\chi \in\left[0, \chi_{1}\right] \mid L(\chi) \leq \rho(\chi)\right\}$. If $\chi_{\mathrm{c}}<\chi_{1}$ then the initial conditions for $\chi_{c} \leq \chi \leq \chi_{1}$ are given by $x_{0}(\chi)=\rho(\chi) e(\chi)$ and $p_{0}(\chi)=\nu(\chi)$, i.e. the surface starts tangently to the cone.

Furthermore, we have to check whether the curve where this surface reaches the upper part of $\partial G_{+}$with $|\chi| \geq \chi_{1}$ has a position with $r \leq \rho(\chi)$. If not, an adaption as explained in $I$ is necessary.

An alternative procedure would be to calculate the backward solution of the Hamilton system in (3.5) with the initial conditions $x_{0}(\chi)=\rho(\chi) \cdot$ $e(\chi)$ for $|\chi| \geq \min \left(\chi_{c}, \chi_{1}\right)$ and starting tangently to the cone. If for certain $\theta, \phi$ several possibilities for the free surface arise, the one with the smallest value of $r$ is valid. 

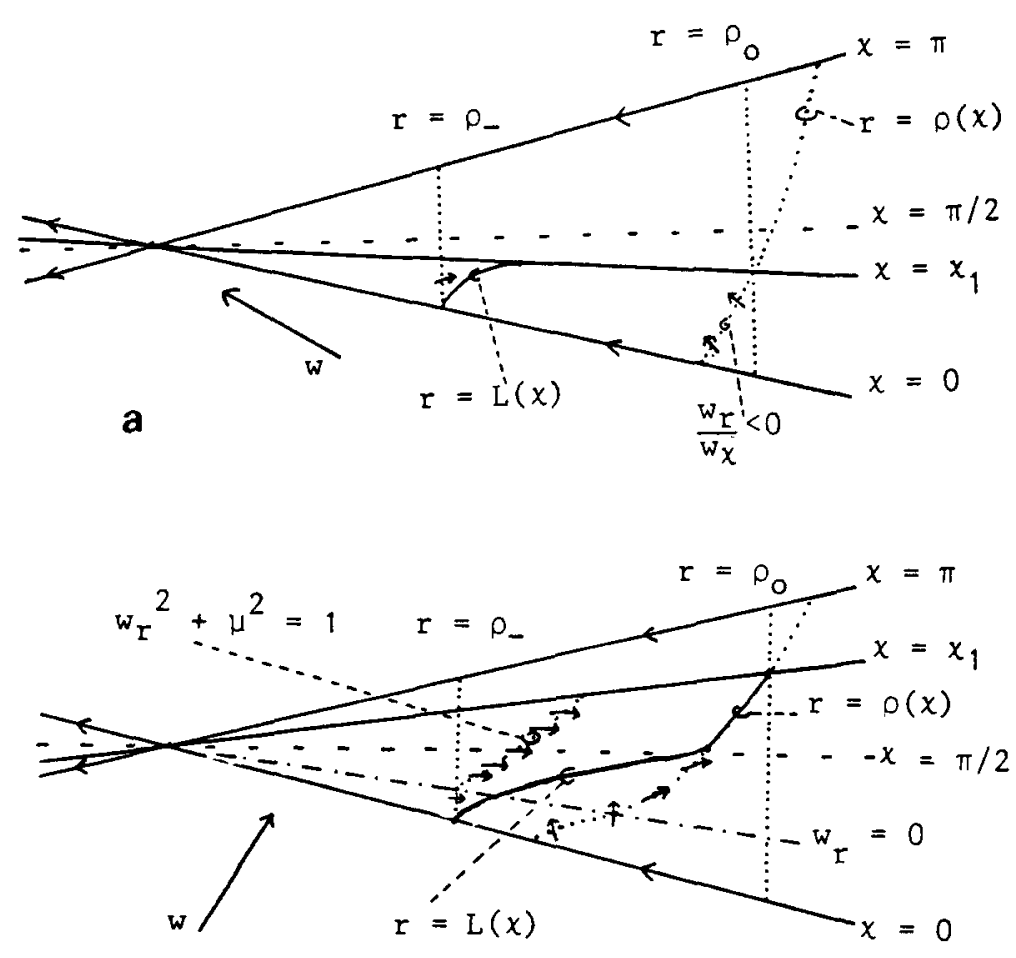

b

Fig. 17.

III: $\theta_{0}+\pi / 2<\alpha \leq \pi$

For $\alpha \uparrow \theta_{0}+\pi / 2$ we observe, that point $\chi_{\mathrm{c}} \downarrow 0$. For $\alpha>\theta_{0}+\pi / 2$ the start curve for the Hamiltonian system is given by

$x_{0}(\chi)=\rho(\chi) \cdot e(\chi)$

for all $|\chi| \in\left[0, \chi_{1}\right]$ if $\alpha<\pi-\theta_{0}$ and for all $|\chi| \in$ $[0, \pi]$ if $\alpha \geq \pi-\theta_{0}$. The surface starts tangently to the cone there with $p_{0}(\chi)=\nu(\chi)$.

As explained before the Hamilton system is also solved backwards in time for $|\chi| \in\left[\chi_{1}, \pi\right]$, if $\alpha<$ $\pi-\theta_{0}$ and the value of $r$ of the free surface at $\theta, \phi$ is the minimal one. Thus it is assured that (3.3.1) is satisfied. Herewith our discussion of the determination of the free surface at the front is rather complete.

Under certain circumstances this surface contains a locus of singular points where the surface makes a corner. For $\alpha=\pi$ there is just one point on the central axis where the surface is singular. For other angles $\alpha$ it is difficult to obtain insight into the structure of this locus using analytic tools.

Let us now consider the construction of the full free surface at the back. On the negative part of the cone $\partial G_{-}$we introduce an angle $\chi$ such that $x$ and $-x$ correspond to the same value $\chi$. Furthermore we introduce the notation $x=-\tilde{L}(\chi) e(\chi)$ with $\tilde{L}>0$ and $e(\chi)$ as in (3.3.5) for the curve the left free surface intersects $\partial G_{-}$. A priori we can say that

$\tilde{\rho}_{-}(\chi) \leq \tilde{L}(\chi) \leq \tilde{\rho}(\chi)$.

Here $\tilde{\rho}_{-}(\chi)>0$ is such that

$\tilde{\mu}\left(-\tilde{\rho}_{-}(\chi) \cdot e(\chi)\right)=-1$.

The function $\tilde{\rho}(\chi)$ describes the largest position where a consistent transition to the rigid boundary 
of the cone can be made, i.e. (see 2.9-10)

$\frac{w}{\|w\|} \cdot \tilde{\nu}(\chi)=\tilde{\mu}(-\tilde{\rho}(\chi) e(\chi))$,

with $\tilde{\nu}(\chi)$ the outward normal on $\partial G_{-}, \tilde{\nu}(\chi)=$ $-\nu(\chi)$ with $\nu$ as in (3.3.5).

$\mathrm{I}_{\mathrm{b}}: 0 \leq \alpha \leq \pi / 2-\theta_{0}$

Initial conditions for the Hamilton system in (3.17) are given by

$x_{0}(\chi)=-\tilde{\rho}(\chi) \cdot e(\chi), \quad p_{0}(\chi)=\tilde{p}(\chi)$.

The surface starts tangently to the cone. If $\alpha>\theta_{0}$ the wind enters for $|\chi|>\chi_{1}$ with $\chi_{1}$ as in (3.3.4). For $|\chi|<\chi_{1},(3.3 .15)$ has to be satisfied. This can be done by solving the Hamilton system for $|\chi|<$ $\chi_{1}$ backwards in time and taking at each $\theta, \phi$ the solution with the smallest norm.

$$
\mathrm{II}_{\mathrm{b}}: \pi / 2-\theta_{0} \leq \alpha \leq \pi
$$

In this case with $\alpha$ close to $\pi / 2-\theta_{0}$ the start curve will consist of two parts, one as in (3.3.15) and one satisfying a condition as in (3.3.8-10)

$-w_{\tilde{\pi}} /\left\|w_{\tilde{\pi}}\right\|=n\left(x_{0}\right), \quad p_{0}(\chi)=w_{\tilde{\pi}}$,

$\left\|w_{\tilde{\pi}}\right\|\|w\|^{-1}=-\tilde{\mu}$,

$(w \cdot \tilde{\tau})^{2}=\|\tilde{\tau}\|^{2}\left(1-\tilde{\mu}^{2}\right)$,

with $w_{\tilde{\pi}}$ the projection of $w$ perpendicular to the curve and $\tilde{\tau}=\mathrm{d} x_{0} / \mathrm{d} \chi$. The reasoning behind (3.3.16) is analogous to the derivation of (3.3.8).

The equation in (3.3.16) must be satisfied as long as characteristics move inwards w.r.t. the cone. Running away from $x_{0}$ along the curve $\gamma(s)$ on the left free surface where $n(\gamma(s))$ lies in the plane $\tilde{\pi}$ corresponding to $x_{0}$ we must have the following properties: (i) $\mu(\gamma(s))$ increases and (ii) $\mathrm{d} n(\gamma(s)) / \mathrm{d} s$ points inwards with respect to the boundary of the cone. It is not simple to check this condition beforehand as in the derivation of (3.3.8). However, in principle one can determine $x_{0}(\chi)$ from (3.3.16) and locally one finds the Hamilton-Jacobi surface $\tilde{\Psi}=0$ with $n=$ $\nabla \tilde{\Psi} /\|\nabla \tilde{\Psi}\|$. Once this has been done, it must be checked whether the test (i), (ii) holds. This test can be reformulated as follows: we check whether with the unit vector $\kappa$ defined by

$\kappa \cdot w_{\tilde{\pi}}=0$,

$\kappa \cdot D^{2} \tilde{\Psi}\left(x_{0}\right) \cdot \tilde{\tau}=0$,

$\kappa \cdot \tilde{\boldsymbol{\nu}}<0$

it is true that

(i) $\nabla \tilde{\mu} \cdot \kappa>0$,

(ii) $\kappa \cdot D^{2} \tilde{\Psi}\left(x_{0}\right) \cdot \tilde{v}$

$$
-\left(\tilde{\nu} \cdot w_{\tilde{\pi}}\right) \cdot\left(w_{\tilde{\pi}} \cdot D^{2} \tilde{\Psi}\left(x_{0}\right) \cdot \kappa\right)<0 .
$$

However, in order to get a qualitative impression of what is happening the original formulation of (i), (ii) suffices.

The condition in (3.3.16) can again be transformed into a concrete ODE. Let us put

$$
\begin{aligned}
\tilde{\chi} & =\pi-\chi, \\
\tilde{e}(\tilde{\chi}) & =e(\pi-\tilde{\chi}), \\
\tilde{w}_{r}(\tilde{\chi}) & =w_{r}(\pi-\tilde{\chi}), \\
\tilde{w}_{\chi}(\tilde{\chi}) & =w_{\chi}(\pi-\tilde{\chi}), \\
x_{0}(\tilde{\chi}) & =-\tilde{L}(\tilde{\chi}) \tilde{e}(\tilde{\chi}),
\end{aligned}
$$

Then we obtain for the function $\tilde{L}(\tilde{\chi})$ with $\tilde{\chi} \geq 0$

$$
\begin{aligned}
\frac{1}{\tilde{L} \sin \theta_{0}} \frac{\mathrm{d} \tilde{L}}{\mathrm{~d} \tilde{\chi}}= & {\left[\tilde{w}_{r} \tilde{w}_{\chi}\right.} \\
& \left.+\sqrt{\left(1-\tilde{\mu}^{2}\right)\left(\tilde{w}_{r}^{2}+\tilde{w}_{\chi}^{2}+\tilde{\mu}^{2}-1\right)}\right] \\
& /\left[\tilde{w}_{r}^{2}+\tilde{\mu}^{2}-1\right]
\end{aligned}
$$

with the initial condition $\left.\tilde{L}\right|_{\tilde{\chi}=0}=\tilde{\rho}_{-}(\pi)$. The sign in front of the square root in this equation follows from an argument as in fig. 16.

In fig. 18 we give an impression of the situation projected on the $x-z$ plane. As usual the solution 


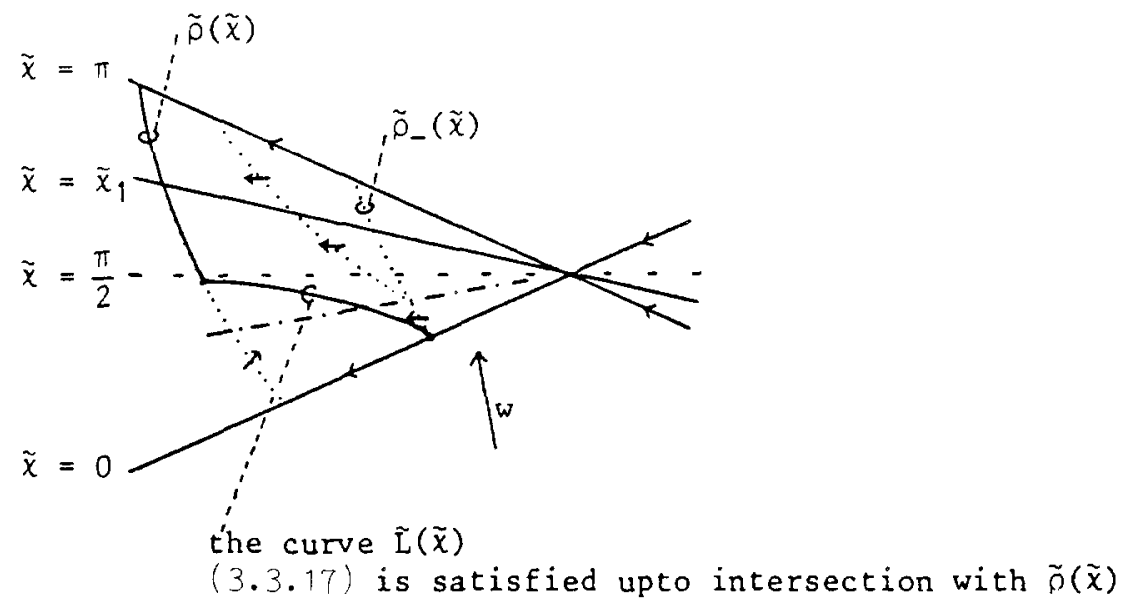

Fig. 18.

of (3.3.19) behaves near $\tilde{\chi}=0$ as

$\tilde{L}(\tilde{\chi})=\tilde{\rho}_{-}(\pi)+\frac{1}{2} C \tilde{\chi}^{2}+\cdots$.

For that constant $C$ one obtains the equation

$$
\begin{aligned}
& \left(\tilde{\boldsymbol{\rho}}_{-}(\pi) \sin \theta_{0}\right)^{-1} C \\
& \quad=w_{r}(\pi)^{-1}\left\{\sin \alpha+\sqrt{\frac{\partial \tilde{\mu}}{\partial \rho} C+\frac{\partial^{2} \tilde{\mu}}{\partial \chi^{2}}}\right\} .
\end{aligned}
$$

When $\alpha$ increases we typically have possibilities as in figs. 19 and 20 . Note that the magical point $\hat{\rho}(\alpha)$ of section 3.1 has now been identified. For $\alpha \rightarrow \pi$ the point $-\hat{\rho}(\alpha) \cdot e(0)$ moves towards $-\hat{\rho}_{-}(0) \cdot e(0)$.

Of course, in the light of (3.3.14) analogous remarks for the construction of the left part of the free surface hold as in $I_{b}$.

\section{On the upperbound for the intensity for a "full plasma solution"}

To conclude this paper we reflect for a moment on the condition

$u>1$ inside the plasma.

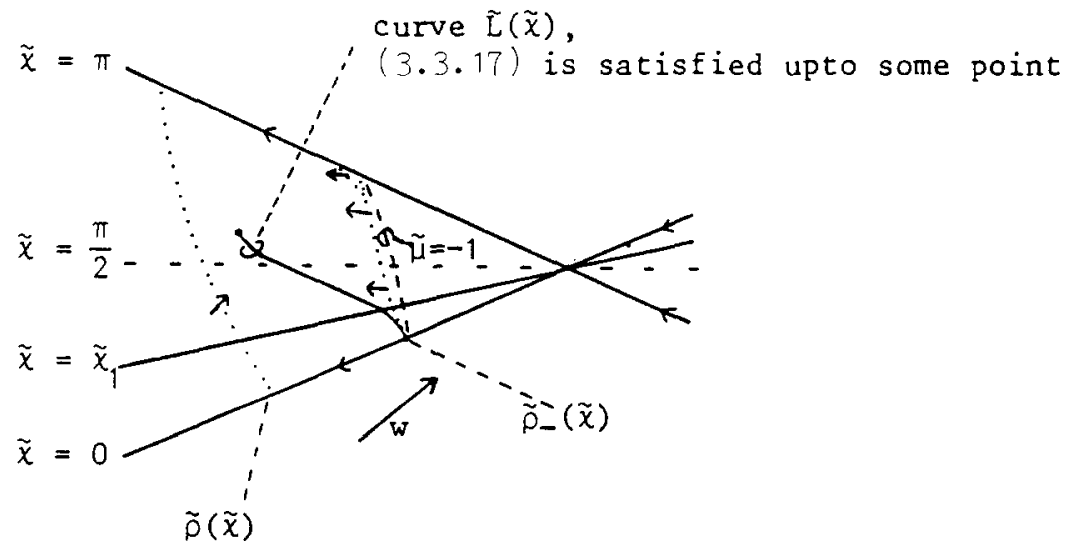

Fig. 19. 


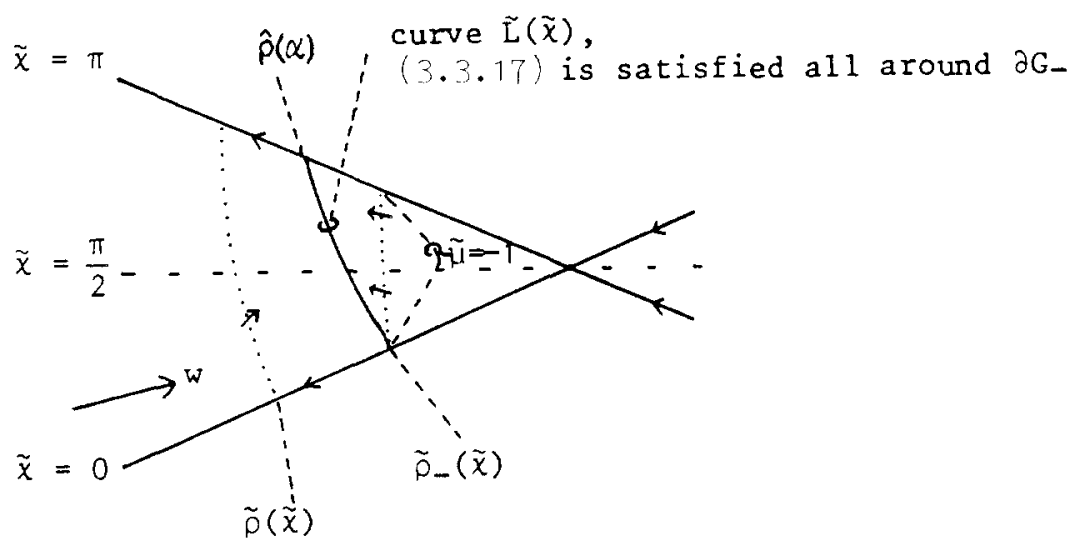

Fig. 20

The constructed approximation must satisfy this condition. If it fails the stationary solution of the full plasma type is just not possible and probably its role is taken over by a more complex, timedependent, pulsating solution, compare [1].

In the situation without wind this occurred when $m>m_{\mathrm{cr}}^{0}$ where the critical value is given by

$m_{\mathrm{cr}}^{0}=34.54+\mathcal{O}\left(a^{2}\right)$.

In the case with wind the critical value will depend on both the strength and the direction of the wind. Let $r=R_{0}(\theta, \phi ; m, w)$ denote the right free surface. If $R_{0}>2$ for some $\theta, \phi$ then the regular approximation in (2.1) will have a minimum at $r=2+\mathcal{O}\left(a^{2}\right)$ for these values of $\theta, \phi$. In this case the critical value $m_{\mathrm{cr}}(w)$ is found as the largest $m$ such that

$$
m \exp \left(-\max _{\theta, \phi \in G} R_{0}(\theta, \phi ; m, w)\right)=\frac{4}{e^{2}}+\mathcal{O}\left(a^{2}\right),
$$

where $G$ is the set of angles inside the cone.
From our construction we deduce that all situations $m_{\text {cr }}(w) \gtreqless m_{0}$ are possible. Moreover, we are led to believe that

i) for a fixed wind strength $m_{\mathrm{cr}}(w)$ will be a decreasing function of $\alpha$ (i.e. the more the wind blows towards the laser the lower $m_{\mathrm{cr}}(w)$ will be).

ii) for a fixed angle $\alpha$ and $\|w\|$ large, $m_{\mathrm{cr}}(w)$ will behave as the solution of

$m \exp \left(-\rho_{ \pm}(m,\|w\|)\right)=\frac{4}{e^{2}}+\mathcal{O}\left(a^{2}\right)$,

with $\rho_{ \pm}=\sqrt{(m / 2)\left(1 \pm\|w\| / \sqrt{4+\|w\|^{2}}\right.}, \quad$ (see (3.3)), where the + is valid for small angles $\alpha$ and the - is valid for $\alpha$ close to $\pi$.

The conclusion is that wind can have a serious impact on the stability of the full plasma solution compared with the case without wind.

\section{References}

[1] W. Eckhaus, A. van Harten and Z. Peradzyński, A singularly perturbed free boundary problem describing a laser sustained plasma, SIAM J. Appl. Math. 45 (1985).

[2] W. Eckhaus, Asymptotic Analysis of Singular Perturbations (North-Holland, Amsterdam, 1979).

[3] R. Courant and D. Hilbert, Methods of Mathematical Physics vol. II (Wiley-Interscience, New York, 1962). 